\title{
Abstracts
}

Pathologe $2020 \cdot 41: 677-690$

https://doi.org/10.1007/s00292-020-00849-x

Online publiziert: 16 . Oktober 2020

(c) Springer Medizin Verlag $\mathrm{GmbH}$, ein Teil von

Springer Nature 2020

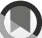

Check for
updates

\section{6th annual congress of the Swiss Society of Pathology}

\author{
November 12-14, 2020
}

\author{
Kultur \& Kongresshaus Aarau
}

Prof. Dr. Rainer Grobholz

Congress President

Prof. Dr. Gieri Cathomas

President SGPath-SSPath

\section{Orals}

\section{1}

Quick Annotator: an open source digital pathology tool for annotating objects 70 times faster than manual annotation Runtian Miao ${ }^{1 *}$, Rob Toth ${ }^{2}$, Anant Madabhushi ${ }^{3}$, Andrew Janowczyk ${ }^{4}$ ${ }^{1}$ Case Western Reserve University, Department of Biomedical Engineering, Cleveland OH, The United States; ${ }^{2}$ Toth Technology LLC, Dover, NJ, The United States; ${ }^{3}$ Case Western Reserve University, Department of Biomedical Engineering, Cleveland OH, The United States; Louis Stokes Veterans Affair Medical Center, Cleveland, $\mathrm{OH}$, The United States; ${ }^{4}$ Case Western Reserve University, Department of Biomedical Engineering, Cleveland $\mathrm{OH}$, The United States; Lausanne University Hospital, Precision Oncology Center, Vaud, Lausanne, Switzerland

Background: Machine learning approaches for the segmentation of histologic primitives (e.g., cell nuclei) in digital pathology (DP) Whole Slide Images (WSI) require large numbers of exemplars. Unfortunately, annotating each object is laborious and often intractable even in moderately

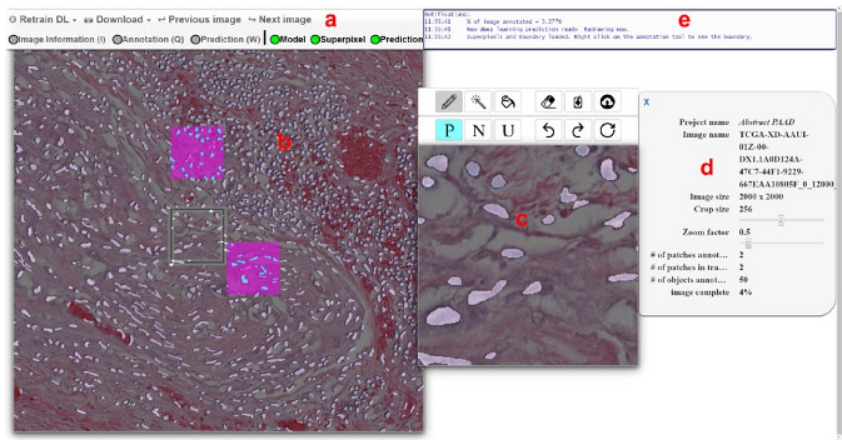

Fig. 1 | $01 \Delta$ User interface of QA: (a) navigation bar with overlay and status indicators; (b) image overview area showing original image, user annotations (region in fuchsia with objects highlighted in blue), and DL predictions (white overlay); (c) annotation window of selected region at higher magnification; (d) image metadata juxtaposed with crop and zoom factor selectors; (e) notification log for status updates

All authors marked with * are the presenting authors.
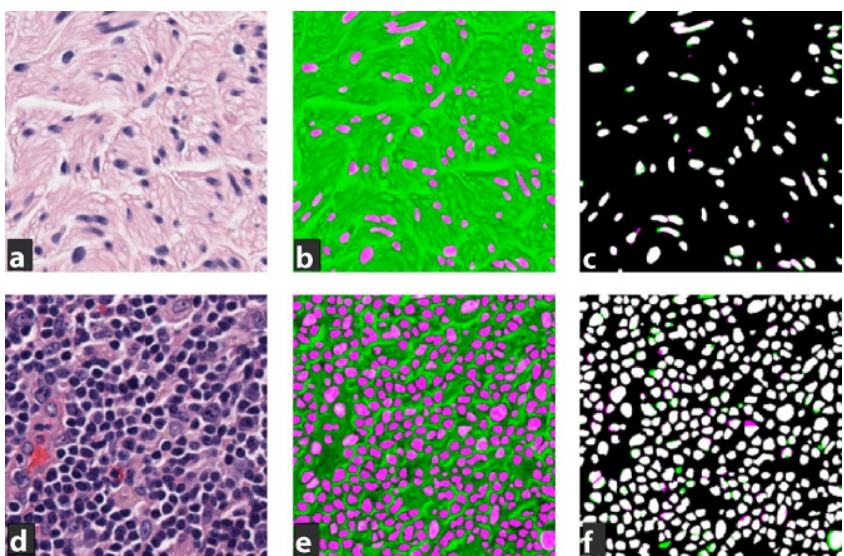

Fig. 2 | 01 A Fig. 2 shows (a, d) original $500 \times 500$ ROls with (b, e) associated annotations overlaid in fuchsia. This manual result is compared with the output from QA (c, f), such that pixels in common between both results are white, and those missing from $(\mathbf{b}, \mathbf{e})$ appear green, and those unique to QA appear pink. Their high concordance, as indicated by the presence of mostly white pixels, is supported by f-scores of 0.99 (top) and 0.96 (bottom). Notably, although highly similar, QA's annotations were produced over 70 times faster than manual efforts

sized cohorts. Here, we present an open source tool, Quick Annotator (QA), designed to improve the annotation efficiency of histologic primitives on WSIs of human annotators by $70 \times$ via the integration of deep learning (DL) and active learning. While the user annotates regions of interest (ROI) via an intuitive web interface, a DL model is concurrently optimized using these annotations and applied to the ROI. The user iteratively reviews DL results to either (a) bulk accept accurately annotated regions, or (b) correct erroneously segmented objects to improve subsequent model suggestions, before transitioning to other ROIs.

Methods: 3 Pancreatic adenocarcinoma (PAAD) TCGA WSIs were employed to evaluate the efficiency improvements afforded by QA. To estimate manual annotation speed, QuPath [1] was used to delineate cell nuclei in $2500 \times 500$ ROIs. QA usage time was recorded for annotating the equivalent of $600500 \times 500$ ROIs, including the manual ROIs (• Fig. 1). Resulting annotations were quantitatively and qualitatively compared. Results: QA employment yielded 345,722 nuclei in $535 \mathrm{~min}$, or 10.8 nuclei per second (NPS). A similar feat employing QuPath necessitates $\sim 685 \mathrm{~h}$ based on the 0.14 NPS performance witnessed in the $500 \times 500$ ROIs. Qualitatively, this $70 \times$ speed improvement does not appear to affect annotation quality (- Fig. 2), which is reflected by a mean pixel-level f-score of 0.975 computed between manual and QA annotation results.

Conclusion: Our preliminary results suggest QA provides a $70 \times$ efficiency improvement while producing annotations highly concordant with manual efforts. The nature of the QA tool further suggests that it could be employed for annotation tasks involving other histologic primitives. 


\section{References}

1. Bankhead P, Loughrey MB, Fernández JA, Dombrowski Y, Mcart DG, Dunne PD, Hamilton PW (2017) QuPath: Open source software for digital pathology image analysis. Scientific Reports 7(1). https://doi.org/10.1038/s41598-017-17204-5

\section{2}

\section{Prevalence of MET Exon 14 mutations or MET amplification in non- small cell lung cancer in Swiss patients}

\section{Spasenija Savic Prince ${ }^{1 *}$, Michel Bihl', Matthias S. Matter', Núria Manuela Zellweger', Sacha I. Rothschild², Lukas Bubendorf'}

'Pathology, Institute of Medical Genetics and Pathology, University Hospital Basel, Basel, Switzerland; ${ }^{2}$ Department of Oncology, University Hospital Basel, Basel, Switzerland

Background: MET-targeted treatment is entering clinical practice for $M E T$ Exon 14-mutated or MET-amplified non-small cell lung cancer (NSCLC). $M E T$ exon 14 skipping mutations occur in $3 \%$ and $M E T$ amplification in 1 to $6 \%$ of unselected NSCLC. They are typically mutually exclusive with other oncogenic driver alterations. The aim of our study was to investigate the prevalence of these $M E T$ alterations in treatment-naïve pre-selected NSCLC from our routine clinical practice, were MET analyses were generally performed sequentially in NSCLC with high MET expression and wild type status for EGFR, KRAS, ALK and ROS1.

Methods: 580 consecutive NSCLC were retrospectively evaluated. High MET expression was defined as complete membranous MET staining with at least moderate intensity in $\geq 50 \%$ of tumor cells. MET exon 14 analysis was performed by Sanger sequencing. MET gene copy numbers were evaluated by fluorescence in situ hybridization (FISH). MET amplification was defined as a $M E T / C E N 7$ ratio of $\geq 2.0$. MET immunohistochemistry (IHC) was performed on histology specimens and cellblocks, but not on conventional cytology preparations. Therefore, prevalence data of MET alterations were analyzed separately for IHC pre-selected and non-preselected NSCLC wild type for other oncogenic driver alterations.

Results: $66 \%$ (302/457) of NSCLC had a high MET expression. There was no difference between the prevalence of MET exon 14 mutations in IHC pre-selected and non-preselected NSCLC (8.3\% (9/99) and 8.1\% (7/86), respectively, $p=1.0)$. In contrast the prevalence of $M E T$ amplification was higher in MET IHC pre-selected NSCLC (11.5\% (11/85) and $3.2 \%(1 / 30)$ respectively, $p=0.29$ ).

Conclusions: MET exon 14 skipping mutations are enriched in NSCLC wild type for other oncogenic driver alterations with the prevalence of $>8 \%$ being higher compared to unselected NSCLC ( $3 \%)$. High MET expression does not enrich for MET exon 14 mutations. However, MET IHC seems to be a cost-effective approach for pre-screening NSCLC for further MET FISH analysis.

\section{3}

\section{The histologic presentation of hepatitis E reflects patients' immune status and pre-existing liver condition}

Daniela Lenggenhager ${ }^{\prime *}$, Samuel Pawel ${ }^{2}$, Hanna Honcharova-Biletska' ${ }^{1}$ Katja Evert ${ }^{3}$, Jürgen J. Wenzel ${ }^{4}$, Matteo Montani ${ }^{5}$, Eva Furrer ${ }^{2}$, Montserrat Fraga ${ }^{6}$, Darius Moradpour ${ }^{6}$, Christine Sempoux ${ }^{7}$, Achim Weber ${ }^{7}$

'Department of Pathology and Molecular Pathology, University of Zurich and University Hospital Zurich, Zurich, Switzerland; ${ }^{2}$ Epidemiology, Biostatistics and Prevention Institute, University of Zurich, Zurich, Switzerland; ${ }^{3}$ Institute of Pathology, University of Regensburg, Regensburg, Germany; ${ }^{4}$ Institute of Clinical Microbiology and Hygiene, Regensburg University Medical Center, University of Regensburg, Regensburg, Germany; ${ }^{5}$ Institute of Pathology, University of Berne, Berne, Switzerland; ${ }^{6}$ Division of Gastroenterology and Hepatology, Centre Hospitalier Universitaire Vaudois, University of Lausanne, Lausanne, Switzerland; ${ }^{7}$ Institut Universitaire de Pathologie, Centre Hospitalier Universitaire Vaudois, University of Lausanne, Lausanne, Switzerland
Background: Hepatitis E virus (HEV) infection is a main cause of acute hepatitis worldwide. Given that, the histopathology of hepatitis $\mathrm{E}$ is relatively poorly characterized, and it is unclear what exactly determines its remarkable variability. Thus, we aimed at a systematic analysis of hepatitis E histology, especially with regard to the clinical setting.

Methods: A large cohort of liver samples $(n=52)$ from 41 patients with molecularly proven hepatitis E (28 HEV genotype (gt) 3, three gt1, one gt 4 and 9 undetermined gt) and very different clinical background, were systematically evaluated for 33 histopathologic features. Following one approach, the samples were assigned to one of five generic histologic patterns. In another approach, they were subjected to hierarchical clustering. The resulting clusters were analyzed for their association with clinical parameters. Results: Eighteen of the 41 (44\%) patients had no known immunosuppression whereas 23 (56\%) were immunocompromised. Five patients had pre-existing liver disease (LD). The histopathologic spectrum ranged from almost normal to acute, chronic and steatohepatitis to subtotal necrosis, distributed across all five generic patterns. Hierarchical clustering, identified three histopathologic clusters ( $\mathrm{C} 1-3)$, which segregated along the immune status and pre-existing LD: $\mathrm{C} 1$ contained mostly patients with preexisting $\mathrm{LD}$; histology mainly reflected the respective $\mathrm{LD}$ without pointing specifically to the additional hepatitis E. C2 contained mostly immunocompetent patients; histology mainly displayed florid hepatitis. C3 contained mostly immunocompromised patients; histology mainly displayed smoldering hepatitis. Accordingly, C1-3 differed regarding their differential diagnoses.

Conclusions: Hierarchical clustering suggests three main groups with distinct histopathologic presentation, indicating biologically different manifestations of hepatitis $\mathrm{E}$. The association of histopathologic changes with the patient's immune status and pre-existing LD plausibly explains the diversity of hepatitis E histopathology, and suggests that these factors are the crucial underlying determinants. We expect our results to improve patient management by guiding the clinico-pathologic diagnosis of hepatitis $\mathrm{E}$.

\section{4}

\section{Two distinct immunopathological profiles in autopsy lungs of COVID-19}

Viktor Hendrik Koelzer ${ }^{*}$, Ronny Nienhold ${ }^{2}$, Yari Ciani ${ }^{3}$, Alexandar Tzankov ${ }^{4}$, Haslbauer Jasmin ${ }^{4}$, Thomas Menter', Nathalie Schwab', Maurice Johannes Henkel ${ }^{4}$, Angela Frank ${ }^{2}$, Veronika Zsikla ${ }^{5}$, Niels Willi', Werner Kempf ${ }^{6}$, Thomas Hoyler ${ }^{7}$, Mattia Barbareschi ${ }^{8}$, Holger Moch ${ }^{9}$, Markus Tolnay ${ }^{4}$, Gieri Cathomas ${ }^{2}$, Francesca Demichelis ${ }^{3}$, Tobias Junt ${ }^{7}$, Kirsten Diana Mertz ${ }^{2}$

'Department of Pathology and Molecular Pathology, University Hospital Zurich, Switzerland; ' 2 Institute of Pathology, Cantonal Hospital Baselland, Switzerland; ' ${ }^{L}$ aboratory of Computational and Functional Oncology, Department for Cellular, Computational and Integrative Biology-CIBIO, University of Trento, Trento, Italy; ${ }^{4}$ Institute of Medical Genetics and Pathology, University Hospital Basel, Basel, Switzerland; ${ }^{5}$ Institut für Pathologie and Molekularpathologie, UniversitätsSpital Zürich, Zürich, Switzerland; ${ }^{6}$ Kempf und Pfaltz Histologische Diagnostik, Zürich, Switzerland; ${ }^{7}$ Novartis Institutes for BioMedical Research (NIBR), Basel, Switzerland; ${ }^{8}$ Anatomia ed Istologia Patologica, Ospedale S. Chiara di Trento, Trento, Italy; ${ }^{9}$ Department of Pathology and Molecular Pathology, University and University Hospital Zürich, Zürich, Switzerland

Coronavirus Disease 19 (COVID-19) is a respiratory disease caused by severe acute respiratory syndrome coronavirus 2 (SARS-CoV-2), which has grown to a worldwide pandemic with substantial mortality. Immune mediated damage has been proposed as a pathogenic factor, but immune responses in lungs of COVID-19 patients remain poorly characterized. Therefore we conducted transcriptomic, histologic and cellular profiling of post mortem COVID-19 ( $n=34$ tissues from 16 patients) and normal lung tissues ( $n=9$ tissues from 6 patients). Two distinct immunopathological reaction patterns of lethal COVID-19 were identified. One pattern showed high local expression of interferon stimulated genes (ISGhigh) and cytokines, high viral loads and limited pulmonary damage, the other pattern showed severely damaged lungs, low ISGs (ISGlow), low viral 
loads and abundant infiltrating activated CD8+ T cells and macrophages. ISGhigh patients died significantly earlier after hospitalization than ISGlow patients. Our study may point to distinct stages of progression of COVID-19 lung disease and highlights the need for peripheral blood biomarkers that inform about patient lung status and guide treatment.

\section{5}

\section{Development of a novel custom next generation sequencing panel to facilitate diagnosis and tumor classification of salivary gland neoplasms}

Sandra N. Freiberger ${ }^{1 *}$, Muriel Brada ${ }^{1}$, Christine Fritz' ${ }^{1}$, Martina A Broglie', Tamara Rordorf', Grégoire B Morand ${ }^{2}$, Niels J Rupp'

'Department of Pathology and Molecular Pathology, University Hospital Zurich, Zurich, Switzerland; ' 2 Department of Otorhinolaryngology, Head and Neck Surgery, University Hospital Zurich, Zurich, Switzerland; ${ }^{3}$ Department of Medical Oncology and Haematology, Zurich, Switzerland

Background: The differential diagnosis and classification of salivary gland neoplasms can often be difficult and challenging. Recently, a range of highly specific molecular aberrations, in particular gene fusions as well as mutations, in different salivary gland tumor entities have been described. However, due to the frequent aberrations in otherwise rarely mutated genes, molecular testing is often expensive and time consuming as more than one test is required to cover all possible alterations. Therefore, we designed a novel RNA-based next generation sequencing panel including 27 genes, specifically involved in salivary gland and odontogenic neoplasms. Methods: We designed a custom next generation sequencing (NGS) panel based on the Archer FusionPlex technology including 27 genes, known to be specifically involved in salivary gland and odontogenic neoplasms. Panel validation was performed on RNA from FFPE material of a variety of different salivary gland neoplasm entities and other tumors with known alterations. The results were compared to the results of our current diagnostic standard for the respective alterations (FISH, NGS, Sanger sequencing). Results: Our customized NGS panel reliably detected all known translocations and mutations using RNA from FFPE tissue. The panel allowed analysis of common alterations in salivary gland tumors including the detection of NTRK1-3 fusions that can be used as therapeutic targets. Furthermore, the panel could successfully be used in one case of a difficultto-classify adenoid-cystic carcinoma mimicking pleomorphic adenoma by detection of a MYBL1-NFIB fusion.

Conclusion: This newly developed, salivary gland neoplasm specific NGS panel reliably detects gene fusions and mutations known to be associated with specific entities. This approach can be a comprehensive, timeefficient and powerful tool to facilitate the diagnosis and classification in difficult cases.

\section{Posters}

\section{P 1}

\section{Retrospective post-mortem SARS-CoV-2 RT-PCR of autopsies with COVID-19 suggestive pathology supports absence of lethal community spread in Basel, Switzerland before February 2020}

Haslbauer Jasmin ${ }^{1 *}$, Valeria Perrina ${ }^{1}$, Matthias S. Matter ${ }^{1}$, Athanassios Dellas ${ }^{2}$, Michael Mihatsch', Alexandar Tzankov ${ }^{3}$

'Pathology, Institute of Medical Genetics and Pathology, University Hospital Basel, Basel, Switzerland; ' 2 Department of Clinical Research, University Hospital Basel, Basel, Switzerland; 'Pathology, Institute of Medical Genetics and Pathology, University Hospital Basel, Basel, Switzerland

Background: The first case of Coronavirus disease 2019 (COVID-19) caused by severe acute respiratory syndrome coronavirus 2 (SARS-CoV-2) was recorded in Hubei in December 2019. However, the extent of early community spread in Central Europe before this period is unknown.

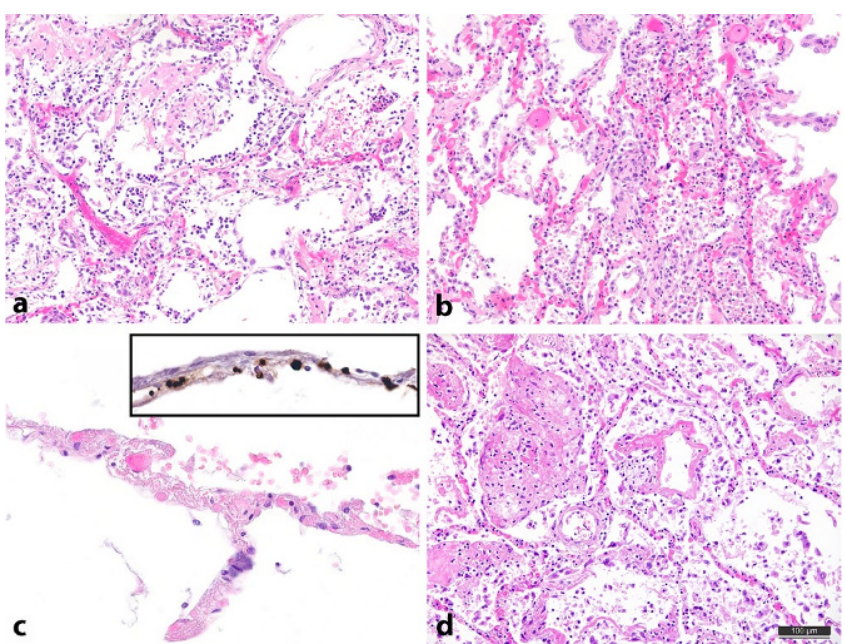

Fig. $1 \mid$ P 1 A

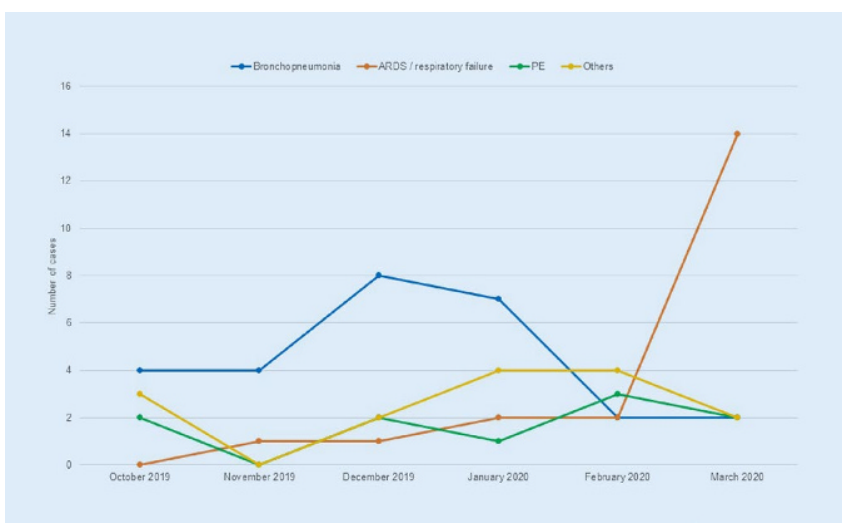

Fig. $2 \mid \mathrm{P} 1 \Delta$

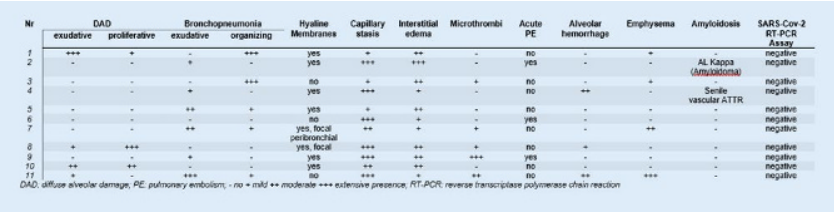

Fig. 3 | P 1 ॥ Pulmonary histological findings and RT-PCR results of adult autopsies with suggestive COVID-19 disease history and histology

A high proportion of asymptomatic cases and undocumented infections, high transmissibility and phylogenetic genomic diversity have engendered the controversial possibility of early international community spread of SARS-CoV-2 before its emergence in China.

Methods: To assess the early presence of lethal COVID-19 in Switzerland, a retrospective analysis of deaths at University Hospital Basel between October 2019 and February $2020(n=310)$ was performed, comparing the incidence of clinical causes of death with March $2020(n=72)$, the month during which the first lethal COVID-19 cases were reported. Trends of COVID-19 suggestive sequelae, such as bronchopneumonia with organization, acute respiratory distress syndrome (ARDS) or pulmonary embolisms (PE) were evaluated. In cases where an autopsy was performed $(n=71)$, analogous analyses were conducted on the cause of death and pulmonary histological findings (• Fig. 1). Eight cases with COVID-19 suggestive clinical history and histopathology between October 2019 and February 2020, and three cases before October 2019 were selected for SARS-CoV-2 RT-PCR. 
Results: A statistically significant rise in pulmonary causes of death were observed in March 2020 ( $p=0.03)$, consistent with the reported emergence of lethal COVID-19 in Switzerland (- Fig. 2). A rise in lethal bronchopneumonia was observed between December 2019 and January 2020, which was likely seasonal. The incidence of lethal ARDS and PE was uniformly low between October 2019 and February 2020. All autopsy cases analyzed by means of SARS-CoV-2 RT-PCR yielded negative results (- Fig. 3).

Conclusion: Our data suggests the absence of early lethal community spread of COVID-19 in Basel before its initial reported emergence in Switzerland in March 2020.

\section{P 2}

\section{An in-depth characterization of cardiac pathology in lethal COVID-19}

Haslbauer Jasmin ${ }^{1 *}$, Alexandar Tzankov', Kirsten Diana Mertz ${ }^{2}$, Nathalie Schwab ${ }^{2}$, Ronny Nienhold ${ }^{2}$, Raphael Twerenbold ${ }^{3}$, Gregor Leibundgut ${ }^{4}$, Anna Katharina Stalder ${ }^{5}$, Matthias S. Matter ${ }^{6}$, Katharina Glatz

'Institute of Medical Genetics and Pathology, University Hospital Basel, Basel, Switzerland; ' 2 nstitute of Pathology, Cantonal Hospital Baselland, Liestal, Switzerland; ${ }^{3}$ Cardiology, University Hospital Basel, University of Basel, Basel, Switzerland; ${ }^{4}$ Cardiology, University Department of Medicine, Cantonal Hospital Baselland, Liestal, Switzerland; ${ }^{5}$ Institute Medical Genetics and Pathology, University Hospital Basel, Basel, Switzerland; ' ${ }^{\text {Pathology, }}$ Institute of Medical Genetics and Pathology, University Hospital Basel, Basel, Switzerland

Background: While coronavirus disease 2019 (COVID-19) primarily affects the respiratory system, detailed pathophysiological changes of the cardiovascular system remain to be elucidated.

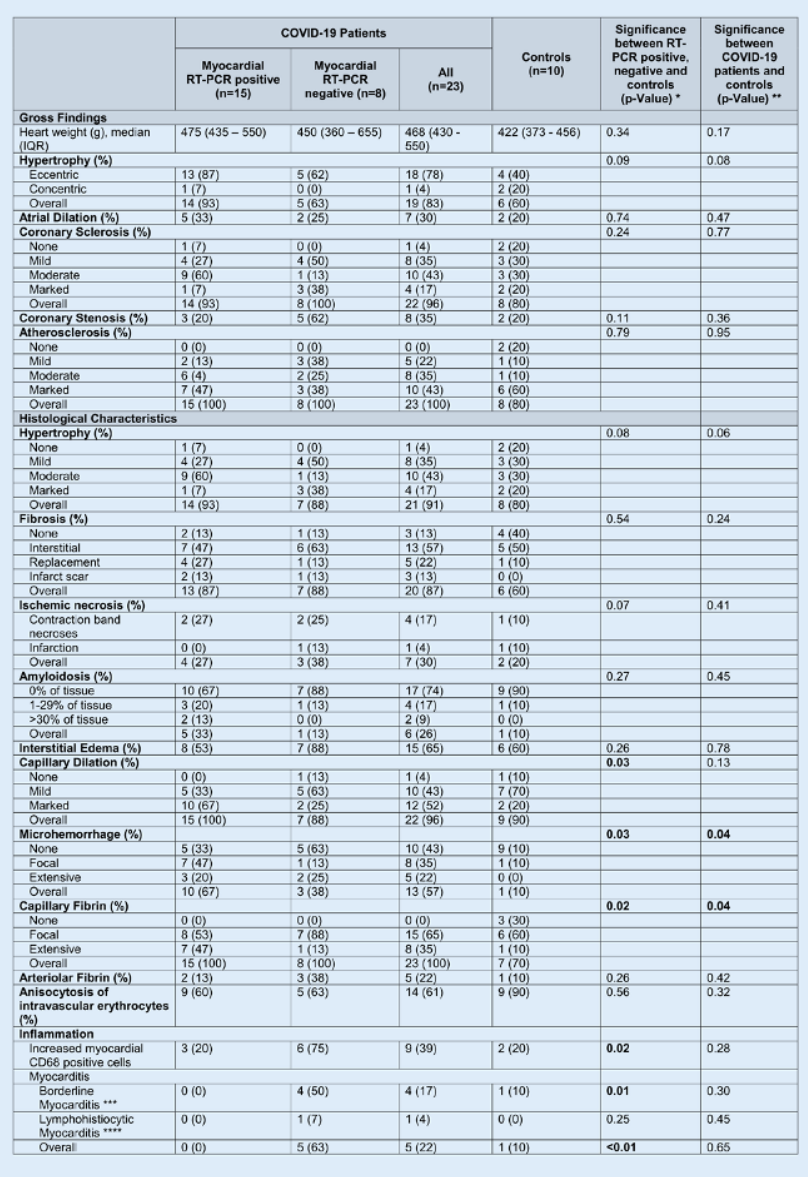

Fig. 2 | P $2 \Delta$ Pathology Findings of Patients with positive and negative SARS-CoV-2 RT-PCR compared to controls

* Significance testing between 3 groups (RT-PCR positive, negative and controls): categorical - Pearson's Chi Square Test for Independence; parametric, continuous - one-way ANOVA; ordinal/non-parametric - Kruskall Wallis one-way ANOVA. $p$-Values considered significant at the 0.05 level ** Significance testing between 2 groups (COVID-19 patients and controls): categorical - Chi Square; parametric, continuous - independent samples t-test; ordinal/non-parametric - Mann Whitney U test. $p$-Values considered significant at the 0.05 level

*** Refer to Materials and Methods for diagnostic criteria of myocarditis 
Methods: Autopsies of 23 patients with COVID-19 were performed at University Hospital Basel and Cantonal Hospital Baselland, Liestal. Representative myocardial tissue sections were collected. Based on Ct-values of myocardial RT-PCR, cases were categorized as SARS-CoV-2 positive $(n=15)$ and negative $(n=8)$. Autopsies of patients without COVID-19 $(n=10)$ with similar clinical sequelae (diffuse alveolar damage, pulmonary thromboembolism, bronchopneumonia) were selected as controls. Histological analysis was performed on $\mathrm{H} \& \mathrm{E}$ and $\mathrm{CAB}$ sections and with immunohistochemistry (CD3, CD20, CD68, Fibrin, ACE2, SARS-CoV-2 N-Protein). Histological characteristics were scored by ordinal and/or categorical grading. The presence of myocarditis was evaluated according to Dallas criteria. Five RT-PCR positive cases underwent in-situ hybridization of viral S- and ORF1AB-RNA. In two cases, electron microscopy was performed. Results: COVID-19 patients had a higher mean age ( $76 \pm 13$ vs. $64 \pm 14$ years, $p=0.04$ ) and increased incidence of hypertension (91 vs. $70 \%$, $p<0.01)$, coronary artery disease (61 vs. $20 \%, p=0.03$ ) and diabetes mellitus (48 vs. $10 \%, p=0.04$ ) than controls (• Fig. 1 ). COVID-19 patients with a positive RT-PCR result died earlier after hospital admission ( $5 \pm 3$ vs. $14 \pm 8$ days, $p<0.01$ ). Increased capillary dilation, capillary fibrin and microhemorrhage were observed in RT-PCR positive myocardium (- Fig. 2); Spearman's Rho analysis revealed a positive correlation amongst these factors (- Fig. 3). All cases fulfilling diagnostic criteria for myocarditis (borderline $n=4$, lymphohistiocytic $n=1$ ) were RT-PCR negative. In 3 cases, in-situ hybridization revealed viral RNA in interstitial cells.

Conclusions: Myocardial capillary dilation, fibrin deposition and microhemorrhage may be the histomorphological correlate of the hypercoagulable state observed in critically-ill COVID-19 patients and are thus suggestive of a generalized vasculopathic component in its pathophysiology. RT-PCR negativity in cases with myocarditis implies a secondary immunological response, which warrants further characterization.

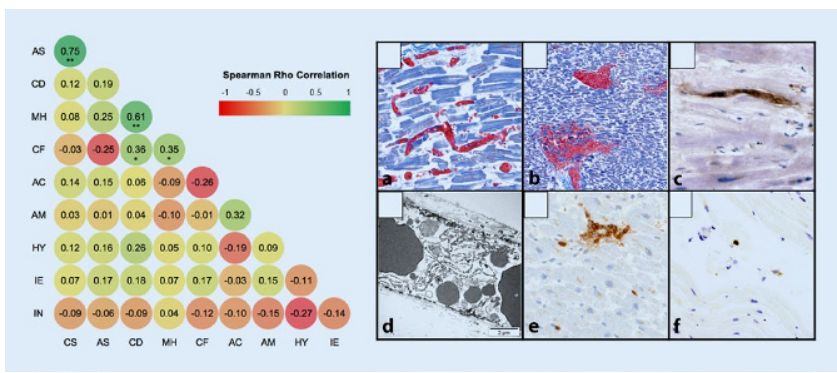

Fig. 3 | P 2 \ Spearman Rho Heat Map of Pathological Characteristics of Myocardium in Lethal COVID-19. Statistically significant correlations between $\mathrm{AS}$ and $\mathrm{CS}, \mathrm{MH}$ and $\mathrm{CD}, \mathrm{CF}$ and $\mathrm{CD}$ and $\mathrm{MH}$ and $\mathrm{CF}$. ${ }^{*}$ Correlations significant at the 0.05 level (2-tailed). ${ }^{* *}$ Correlations significant at the 0.01 level (2-tailed). a: Marked capillary dilation (CAB, 400x); b: Hyperemic intramyocardial vessels with parenchymal microhemorrhage (CAB, 100x); c: Fibrin thrombus in an intramyocardial capillary (fibrin IHC, 400x); d: Capillary vessel containing irregularly shaped and anisocytotic erythrocytes coated by a thin layer of fibrin. Fibrin deposits along the vessel wall and intraluminal fibrin threads (electron microscopy); e: Lymphocytic borderline myocarditis: Collection of T-lymphocytes without myocardial necrosis (CD3, 400x); f: Viral genome of SARS-CoV2 in interstitial cells, probably mononuclear inflammatory cells (lymphocyte/macrophage) (SARS-CoV2 ISH, 400x)

\section{P 3}

\section{SARS-CoV-2 virus detection in tissues by reverse transcriptase quantitative PCR: set-up of a methodology suitable for FFPE samples}

Karine Lefort ${ }^{1 *}$, Damien Maison ${ }^{2}$, Sabina Berezowska ${ }^{2}$, Nathalie Piazzon ${ }^{2}$, Laurence de Leval', Bettina Bisig'

'Deparment of Laboratory Medicine and Pathology, Institute of Pathology, Lausanne University Hospital and Lausanne University, Lausanne, Switzerland; ' ${ }^{2}$ epartment of Laboratory Medicine and Pathology, Institute of Pathology; Lausanne University Hospital and Lausanne University, Lausanne, Switzerland

Background: In the context of the COVID-19 pandemic, pathology departments face the need to develop sensitive assays to detect SARS-CoV-2 virus in formalin-fixed paraffin-embedded (FFPE) tissue samples, both from autopsies and from surgical and bioptic specimens. We describe the methodology set up at the University-Institute of Pathology in Lausanne. Methods: Total RNA was extracted from FFPE samples from lung, liver and heart of 12 patients autopsied with intra-vitam confirmed COVID-19. One-step reverse transcriptase quantitative PCR (RT-qPCR) was performed for SARS-CoV-2 mRNAs (E and RdRP genes) as well as for human MSTN as internal control of RNA quality, using commercially available assays (Roche). Specific in vitro-transcribed RNA standards were used in each experiment for determination of viral copy numbers.

Results: Optimal RNA input was 50-250 ng per reaction. Limit of detection of $\mathrm{E}$ gene and RdRP was 7 and 27 copies per reaction, respectively (as determined by Probit analysis). SARS-CoV-2 was detected in 12/12 lung, 6/11 liver and 6/11 heart FFPE samples (one liver sample and one heart sample had an inadequate internal control), with very variable virus levels. The highest amounts were detected in the lung (range E gene: 11537,000 copies per reaction; RdRP: 0-64,345), followed by heart (E gene: 0-9756; RdRP: 0-129) and liver (E gene: 0-583; RdRP: 0-9). All samples found positive for $\mathrm{E}$ gene were also positive for RdRP, but not reciprocally. Conclusions: We show that a commercially available kit can be used for sensitive and quantitative SARS-CoV-2 virus detection in FFPE tissues. The limit of detection was lower for E gene than for RdRP. A suitable internal control for RNA quality is highly recommended in order to rule out false negative results.

\section{P 4}

Mutational landscape of marginal zone B-cell lymphomas of various origin: organotypic pathways and diagnostic potential for organ assignement

Visar Vela", Darius Juskevicius, Thomas Menter, Vincent Pillonel, Stefan Drinhofer, Alexandar Tzankov

Pathology, Institute of Medical Genetics and Pathology, University Hospital Basel, University of Basel, Basel, Switzerland

Background: Marginal zone lymphomas (MZL) comprise nodal (NMZL), splenic (SMZL), and extranodal (EMZL) instances, accounting for 10, 15 and $75 \%$, respectively, and, as a whole, for $12 \%$ of all B-cell neoplasms. MZL have no disease-defining phenotype and the diagnostic borders to other lymphomas are blurred. MZL are known to bear typical site-of-orgin translocations, yet their mutational landscape has not been integratively analyzed.

Principals/Methodology: Gene mutation data of 916 MZL, namely 287 ocular adnexal (OA), 93 pulmonary (P), 71 salivary gland (SG), 59 gastric (G), 38 cutaneous, 18 thyroid (T), 15 dura matter (D), 115 NMZL and 220 SMZL were retrieved from our own digital archives and published other groups' studies. Data were pooled and used for inter-entity comparison. Number and frequency of mutated cases ( $\geq 4$ and/or $\geq 5 \%$ /entity), were set to generate a gene shortlist. Statistical differences were calculated by the two-tailed Fisher's exact test; P-values were corrected for multiple testing $(p<0.005)$.

Results: Out of 223 genes, mutational data in 11 appeared to be of importance respecting MZL origin. NMZL more commonly displayed mutations 
in KMT2D, FAS, TNFRSF14 or EZH2 compared to SMZL, in KMT2D or TNFRSF14-compared to OAMZL, and in KMT2D only-compared to SGMZL. Mutations in NF1 were conjoined to GMZL compared to PMZL and DMZL. TMZL more often harbored mutations in TET2 compared to PMZL, SGMZL and NMZL. In PMZL, mutations in KMT2D or B2M were more frequent than in OAMZL. OAMZL more commonly harbored mutations in TNFAIP3 than NMZL and GMZL. Finally, in DMZL mutations in TNFAIP3 were more common than in SMZL.

Conclusion: Mutations of distinct genes show origin-preferential distribution among MZL. Recognition of such mutational distribution patterns may be of potential help assigning MZL origin in difficult cases and possibly also pave the way for novel tailored treatment concepts.

\section{P 5}

\section{Quality comparison of computer-assisted T-cell and tumor-bud detection in pT1 colorectal cancer from four different pathology institutions*}

Linda Studer ${ }^{*}$, John-Melle Bokhorst ${ }^{2}$, Inti Zlobec ${ }^{3}$, Alessandro Lugli ${ }^{4}$, Andreas Fischer ${ }^{1}$, Francesco Ciompi ${ }^{2}$, Jeroen van der Laak ${ }^{2}$, Iris Nagtegaal ${ }^{2}$, Heather Dawson $^{4}$

${ }^{1} \mathrm{i} C \mathrm{~S}$ Sys, Hochschule für Technik und Architektur Freiburg, Freiburg, Switzerland; ${ }^{2}$ Radboud University Medical Center, Nijmegen, Netherlands; ${ }^{3}$ Translational Research Unit, University of Basel, Basel, Switzerland; ${ }^{4}$ Institute of Pathology, University of Bern, Bern, Switzerland

Background: Tumor budding and T-cell infiltrates are robust prognostic biomarkers in colorectal cancer. The identification of tumor buds and Tcells can be expedited and facilitated using deep learning. However, due to factors such as pre-analytics, universal tools must be validated rigorously. The aim of this study was to verify and compare the quality of automated detection of tumor buds and T-cells in a heterogeneous slide collection.

Methods: We validated deep learning algorithms for tumor budding and CD8+ T-cell detection on double-stained immunohistochemical slides

\begin{tabular}{lccc}
\hline \hline Institution & \# of Slides & Precision & Recall \\
\hline A & 44 & $61.1 \%$ & $53.5 \%$ \\
\hline B & 10 & $58.7 \%$ & $38.0 \%$ \\
\hline C & 8 & $59.4 \%$ & $23.2 \%$ \\
\hline D & 14 & $73.1 \%$ & $43.5 \%$ \\
\hline Overall & 76 & $61.6 \%$ & $48.6 \%$ \\
\hline \hline
\end{tabular}

Fig. 1 | P $5 \Delta$ Overview of the quality control results for the automated tumor bud detection

\begin{tabular}{lccc}
\hline \hline Institution & \# of Slides & Precision & Recall \\
\hline A & 44 & $88.1 \%$ & $92.3 \%$ \\
\hline B & 10 & $89.4 \%$ & $92.9 \%$ \\
\hline C & 8 & $96.1 \%$ & $98.4 \%$ \\
\hline D & 14 & $90.9 \%$ & $91.9 \%$ \\
\hline Overall & 76 & $89.9 \%$ & $93.0 \%$ \\
\hline \hline
\end{tabular}

Fig. 2 | P $5 \Delta$ Overview of the quality control results for the automated T-cell detection
(AE1-AE3 and CD8) from our pT1 colorectal cancer cohort from 4 different institutions, originating from 1992-2017. At least $20 \%$ of slides from each institute (total 76) were randomly selected for quality control. Staining and scanning was performed in a single laboratory using the same protocol. For comparison, a single observer identified tumor buds within the tumor budding hotspot $\left(0.785 \mathrm{~mm}^{2}\right)$ as well as correcting the T-cell algorithm output.

Results: - Fig. 1 shows the number of correctly identified tumor buds, - Fig. 2 the T-cell detection. The most consistent performance is achieved on slides from Institute A. Notably, institution C, which achieves the best results for Tcell detection shows the worst results for tumor bud detection. Upon review, heterogeneity in staining intensity between slides and within the same slide emerged as a major factor for tumor buds missed by the algorithm.

Conclusion: The algorithm for T-cell detection achieves better results for all institutions in comparison to tumor bud detection. This suggests that different algorithms face unique challenges in detecting individual objects. T-cell detection appears relatively robust, whereas in addition to known challenges in high inter- and intra-observer variability, tumor bud detection is more prone to pre-analytical factors. A variety of approaches can be used to address these issues in the development and validation of automated detection methods.

* Student paper

\section{P 6}

\section{PatchSorter: a high throughput digital pathology tool for cell labeling}

Tasneem Talawalla', Anant Madabhushi', Andrew Janowczyk ${ }^{3 *}$

${ }^{1}$ Case Western Reserve University, Department of Biomedical Engineering,

Cleveland OH, USA; ${ }^{2}$ Case Western Reserve University, Department of Biomedical Engineering, Cleveland OH, USA; Louis Stokes Veterans Affair Medical Center, Cleveland, $\mathrm{OH}, \mathrm{USA} ;{ }^{3}$ Case Western Reserve University, Department of Biomedical Engineering, Cleveland OH, USA; Lausanne University Hospital, Precision Oncology Center, Vaud, Lausanne, Switzerland

Background: Recently significant interest in development of computational pathology tools for diagnosis, prognosis, and therapy response of many diseases has emerged. These approaches typically rely on accurate understanding of both location and cell type present within histology slide images. While image analysis algorithms can identify the location of millions of cells on digitized pathology images, a subsequent step is needed to identify cell type. Manually assigning labels (e.g., inflammatory, epithelial) to each cell individually remains an intractable task. Here, we present PatchSorter, a browser-based high-throughput cell labeling tool which enables the user to review and assign labels at a group, as opposed to individual cell level, thus greatly improving labeling efficiency. As the user classifies groups of cells, PS's deep learning component iteratively increases separation between target cell groups within a low dimensional representational space (• Fig. 1), thus further improving labeling efficiency.

Methods: A random subset of 14,330 cells, with ground truth labels indicating membership in either inflammatory or epithelial classes, from the multi-organ $27 \mathrm{H} \& \mathrm{E}$ image Hover-net dataset [1] was used to evaluate PS. To estimate manual labeling speed, a Photoshop clone (Gimp) was used to label 120 cells from a representative image. Labels generated from PS were quantitatively compared against provided inflammatory or epithelial class labels using the accuracy metric.

Results: Labeling 14,330 cells using PS required 9607 s, yielding 1.5 labels per second (LPS). The estimated time to perform the same task manually would take $23,883 \mathrm{~s}$, based on the .6 LPS recorded when manually using Gimp. Qualitatively, this $60 \%$ speed improvement only modestly affects labeling fidelity, as indicated by a $93 \%$ accuracy between PS produced labels and ground truth.

Conclusion: PatchSorter provides a $60 \%$ efficiency improvement for assigning cells to appropriate cell-type, while also yielding a $93 \%$ accuracy. Additional, independent validation and user testing is warranted. 


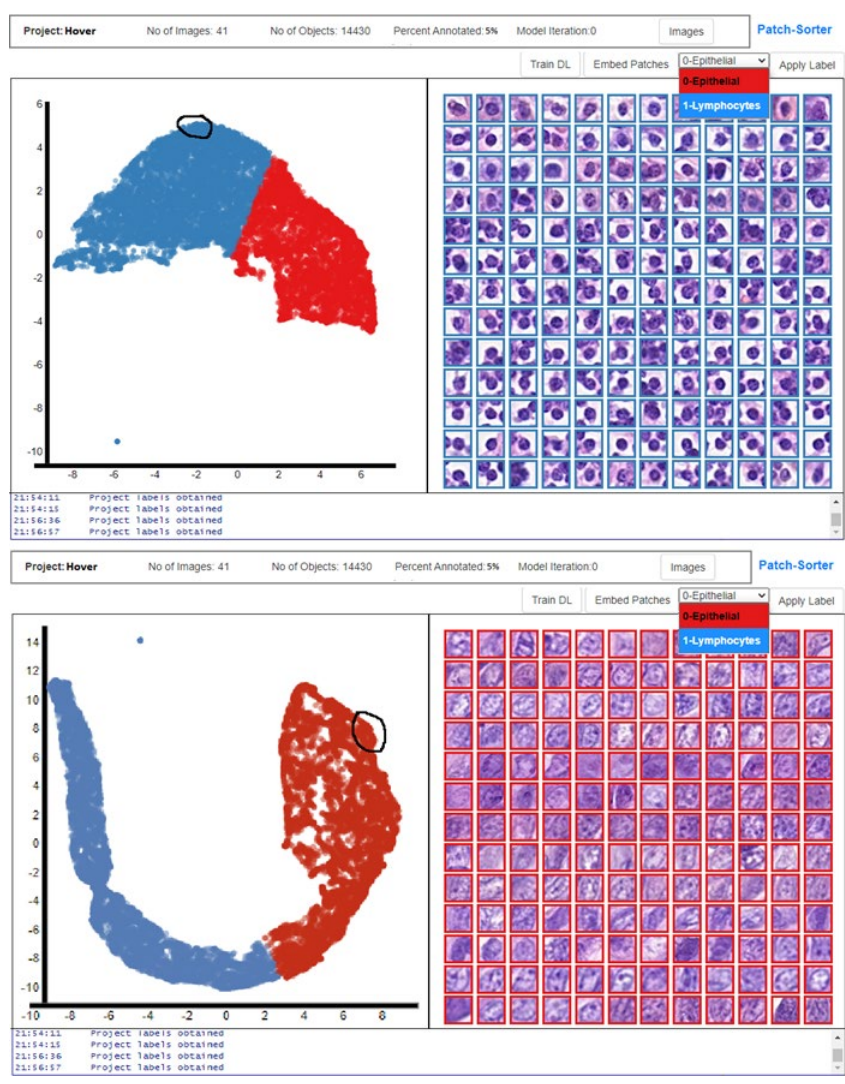

Fig. 1 | P $6 \Delta$ (Top) To begin, the user lassos points of interest in the Cartesian plot (left) which are subsequently presented for review and bulk labeling (right). (Bottom) As labeling progresses, the feature space is improved, further purifying and separating predicted groups in the plot to facilitate improved efficiency in labeling

\section{References}

1. S. Graham, Q. D. Vu, S. E. A. Raza, A. Azam, Y-W. Tsang, J.T. Kwak and N. Rajpoot. „HoVer-Net: Simultaneous Segmentation and Classification of Nuclei in MultiTissue Histology Images." Medical Image Analysis, Sept. 2019.

\section{P 7}

\section{Mutational profiles of primary pulmonary adenocarcinomas and paired brain metastases}

Sabina Berezowska ${ }^{*}$, Elham Kashani ${ }^{2}$, Philipp Zens ${ }^{3}$, Alexandra Kündig ${ }^{2}$, Christian Fung ${ }^{4}$, Amina Scherz ${ }^{5}$, Evelyne Herrmann ${ }^{6}$, Ekin Ermis ${ }^{6}$, Ralph A. Schmid ${ }^{7}$, Erik Vassella ${ }^{2}$

'Deparment of Laboratory Medicine and Pathology, Institute of Pathology, Lausanne University Hospital and Lausanne University, Lausanne, Switzerland; ${ }^{2}$ Institute of Pathology, University of Bern, Switzerland; ${ }^{3}$ Institute of Pathology, University of Bern, Bern, Switzerland; ${ }^{4}$ Department of Neurosurgery, Inselspital University Hospital Bern, Bern, Switzerland; ${ }^{5}$ Department of Medical Oncology, Inselspital University Hospital Bern, Bern, Switzerland; ${ }^{6}$ Department of Radiation Oncology, Inselspital University Hospital Bern, Bern, Switzerland; ${ }^{7}$ Department of General Thoracic Surgery, Inselspital University Hospital Bern, Bern, Switzerland

Background: Brain metastases (BM) are frequent and clinically relevant in lung cancer patients. Molecular data on paired BM and primary lung cancer is scarce.

Methods: Consecutive solid cancer BM diagnosed at the Institute of $\mathrm{Pa}$ thology Bern from 2000 to 2015 were retrospectively assembled. Paired tissue from pulmonary adenocarcinomas and BM was analyzed by next generation sequencing (NGS; oncomine comprehensive cancer panel v3). Results were compared to routine diagnostics and the TCGA dataset.

Results: NGS was successful in 54/57 consecutive tumor pairs accessible for molecular analysis. Mutation frequencies were in the range expected from TCGA. Primary tumors showed most frequently TP53 missense and truncating mutations (33/55, $60 \%)$, activating KRAS mutations and amplifications (32/55, $58 \%)$, MYC mutations and amplifications $(9 / 55,16 \%)$ and STK11 missense and truncating mutations (9/55, $16 \%$ ). Significantly more KRAS mutations were detected at primary sites compared to in-house data from routine diagnostics and the TCGA (KRAS G13C mutation; $p<0.02$ ). Most genetic alterations were preserved in the BM. Oncoprint analyses revealed a bias towards the EGFR signaling pathway in alterations private to BM, notably, secondary KRAS (22\%), NF1 (9\%), MET (6\%) and MYC alterations (13\%).

Five models of progression emerged from the genetic alterations: Synchronous $\mathrm{BM}(10 / 54 ; 19 \%)$, showed the same set of mutations as the primary sites $(\mathrm{P}=\mathrm{BM})$. Private alterations were seen in $12 / 54(22 \%)$ primary tumors (P>BM, most frequently RICTOR) and 14/54 (26\%) BM, without enrichment of genes $(\mathrm{P}<\mathrm{BM}) .14 / 54(26 \%)$ patients had shared and private mutations at both sites (PBM). Frequent private BM mutations were additional KRAS mutations/copy number gains $(21 \% / 21 \%)$. Only one case showed private KRAS alterations in the primary tumor.

4/54 (7\%) tumor pairs had no shared mutations (P/BM), with different variants of KRAS and TP53.

Conclusions: Our data point to an important role of KRAS in the pathobiology of BM.

\section{P 8}

\section{Putative malignant pleural mesothelioma in situ (MPMIS) with sequential acquisition of genomic alterations on fluorescence in situ hybridization (FISH) examination}

Simon Haefliger ${ }^{*}$, Spasenija Savic Prince', Heinz Borer ${ }^{2}$, Julien Rebetez', Lukas Bubendorf

'Pathology, Institute of Medical Genetics and Pathology, University Hospital Basel, Basel, Switzerland; ${ }^{2}$ Bürgerspital Solothurn, Solothurn, Switzerland

Background: The existence of a malignant mesothelioma (MPMIS) in situ has long been postulated. Here, we present a case of a 57 -year-old man with initial presentation as a putative MPMIS evolving to a diffuse malignant pleural mesothelioma (MPM) during an interval of 8 months. Repeated FISH examination on pleural effusion from both lesions identified sequential acquisition of genomic alterations. Interestingly, in the initial pleural effusion, a substantial number of the mesothelial cells were encircled by lymphocytes, which we refer to as satellitosis.

Methods: Immunochemistry (IC) as well as multiprobe FISH assay were performed on the cytological specimens of both, the putative MPMIS as well as on the diffuse MPM. Further, in order to determine the incidence of satellitosis of mesothelial cells by lymphocytes, we reviewed 60 cases from our archive with proven MPM and 60 cases with the diagnosis of "reactive mesothelial cells".

Results: IC analysis-loss of BAP1 and positivity for calretinin-confirmed the mesothelial and neoplastic nature in both specimens (• Fig. 1). Further, FISH analysis showed homozygous deletion of 9p21 in the initial specimen and unbalanced polysomy of the chromosomes 3,7 , and 17, in addition to the loss of 9p21 in the second specimen (- Fig. 1). In the cases reviewed, "satellitosis" was identified in a total of 5 cases, 2 cases with MPM, and 3 cases with reactive mesothelial cells (- Fig. 2).

Conclusions: FISH analysis can serve as a screening tool to detect MPMIS in patients presenting with no clinical evidence of mesothelioma. Genomic transition from a diploid to an aneuploid state might play a role in progression from MPMIS to invasive MPM. Satellitosis of mesothelial cells by lymphocytes in pleural effusion cytology is a rare and not tumor-specific phenomenon with unclear relevance. 


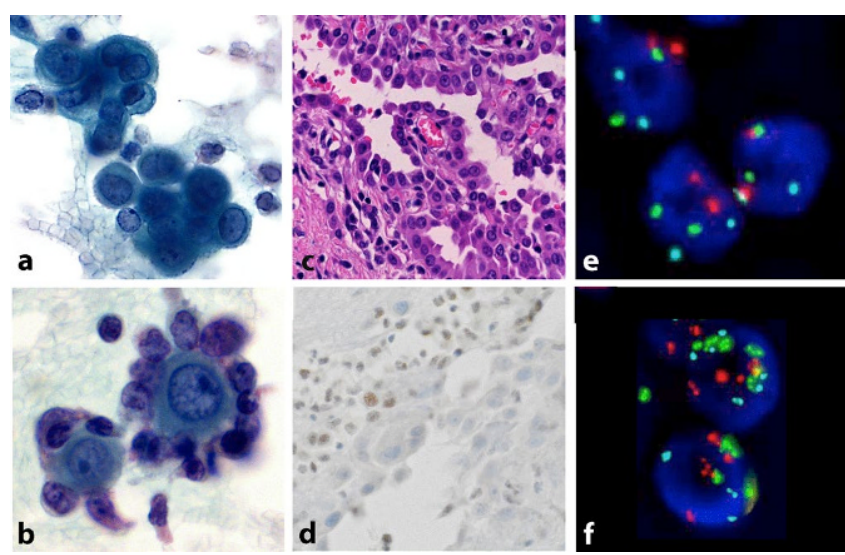

Fig. 1 | P $8 \Delta$ a Pleural effusion cytology from the MPM with large clusters of unequivocal malignant mesothelial cells with prominent nucleoli (Papanicolaou [Pap] stain, x630). b Initial pleural effusion highlighting satellitosis of the mildly atypical mesothelial cells by lymphocytes (Pap stain, x630). c,d Initial pleural biopsy: atypical mesothelial cells on histology (c hematoxylin and eosin stain, $x 400$ ) with loss of BAP1 expression. Internal positive control with nuclear BAP1 expression is observed in the adjacent leucocytes ( $\mathbf{d} \times 400$ ). e Multiprobe FISH of the initial effusion: regular disomic pattern for chromosomes 3 (red), 7 (green), and 17 (blue) and homozygous deletion of the chromosomal region 9p21 (lack of gold signal) (x630). $f$ Multiprobe FISH of the pleural biopsy of malignant mesothelioma 8 months later: homozygous deletion of the chromosomal region 9p21 (lack of gold signal) and polysomy of the chromosome 3 (red), 7 (green), and 17 (blue) in the cytological specimen (f, DAP!)

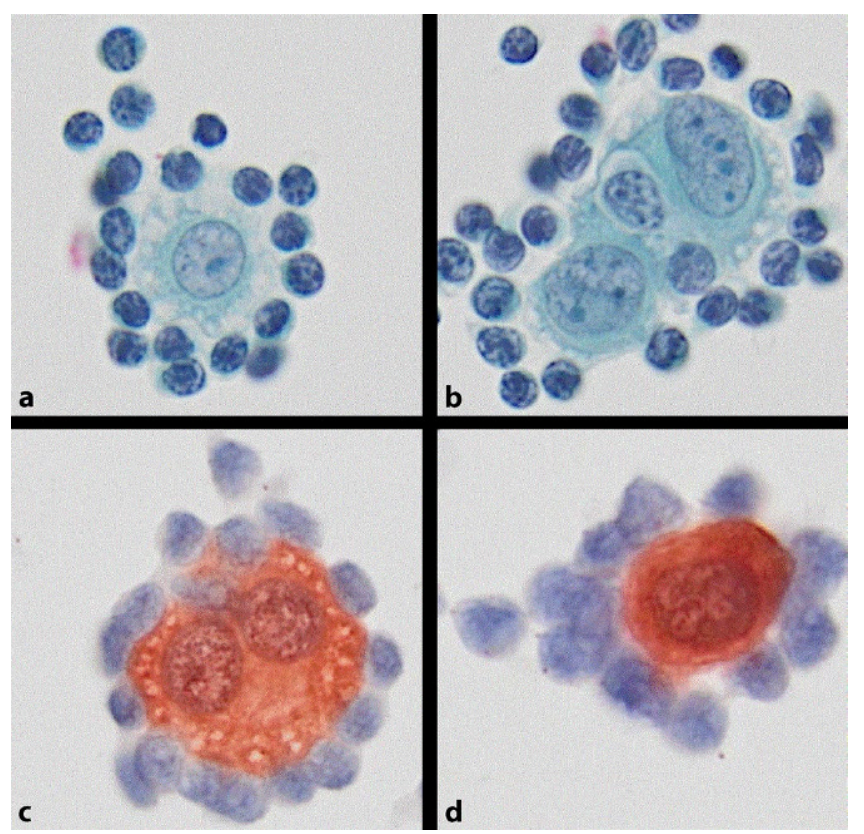

Fig. 2 | P $8 \Delta$ a,b Conventional cytology with benign mesothelial cells surrounded by lymphocytes satellitosis of mesothelial cells by lymphocytes ( $\mathbf{a}, \mathbf{b} \times 630$, Papanicolaou stain). $\mathbf{c}, \mathbf{d}$ The mesothelial nature of the encircled cells was confirmed by immunocytochemistry for calretinin (c,d x630, immunoperoxidase)

\section{P 9}

Progressive disease in sentinel-negative melanoma patients: Biological differences and importance of sentinel lymph node biopsy

Anna Conrad ', Umberto Maccio ${ }^{\prime *}$, Michael Reinehr', David Holzmann ${ }^{2}$, Joanna Mangana ${ }^{3}$, Miriam Wanner', Martin Huellner ${ }^{5}$, Raymond Barnhill, Claire Lugassy ${ }^{6}$, Nicole Lindenblatt ${ }^{7}$, Daniela Mihic-Probst ${ }^{1}$

'Department of Pathology and Molecular Pathology, University Hospital Zurich, Zurich, Switzerland; '2Department of Otorhinolaryngology, Head and Neck Surgery, University Hospital Zurich, Zurich, Switzerland; ${ }^{3}$ Department of Dermatology, University Hospital Zurich, Zurich, Switzerland; ${ }^{4}$ Cancer Registry Zurich and Zug, University Hospital Zurich, and Epidemiology, Biostatistics and Prevention Institute, Zurich, Switzerland; ${ }^{5}$ Department of Nuclear Medicine, University Hospital Zurich, Switzerland; ${ }^{6}$ Departments of Pathology and Translational Research, Institute Curie, Paris, France; ${ }^{7}$ Department of Plastic Surgery and Hand Surgery, University Hospital Zurich, Zurich, Switzerland

Background: Among the most important prognostic factors in melanoma is the sentinel lymph node (SLN) status.

Methods: Using our electronic database we identified 109 of 890 SLN-negative patients with progressive disease (PD). These patients were characterized for melanoma type, molecular type, sequence and extent of metastatic spread. Results: A total of 61 of 109 SLN-negative patients had PD in the SLN-basin indicating false-negative SLN (group-1). 48 of 109 patients had PD at distant sites and were therefore impossible to be identified using SLN biopsy (group-2). Despite distant spread these patients had significantly more single organ metastasis $(p<0.001)$ and significantly longer disease-freesurvival ( $p=0.001)$ compared to group-1. Additionally, to significant differences on a molecular basis between the two groups $(p=0.01)$, all lentigo maligna and spindle-cell-melanomas belonged to group- 2 and all, except one lentigo maligna melanoma, had single visceral metastasis.

Conclusion: Two different biological groups among SLN-negative patients with PD were demonstrated. Extravascularmigratory-metastasis, rather than hematogenous spread, might be responsible for the observed PD with single organ involvement.

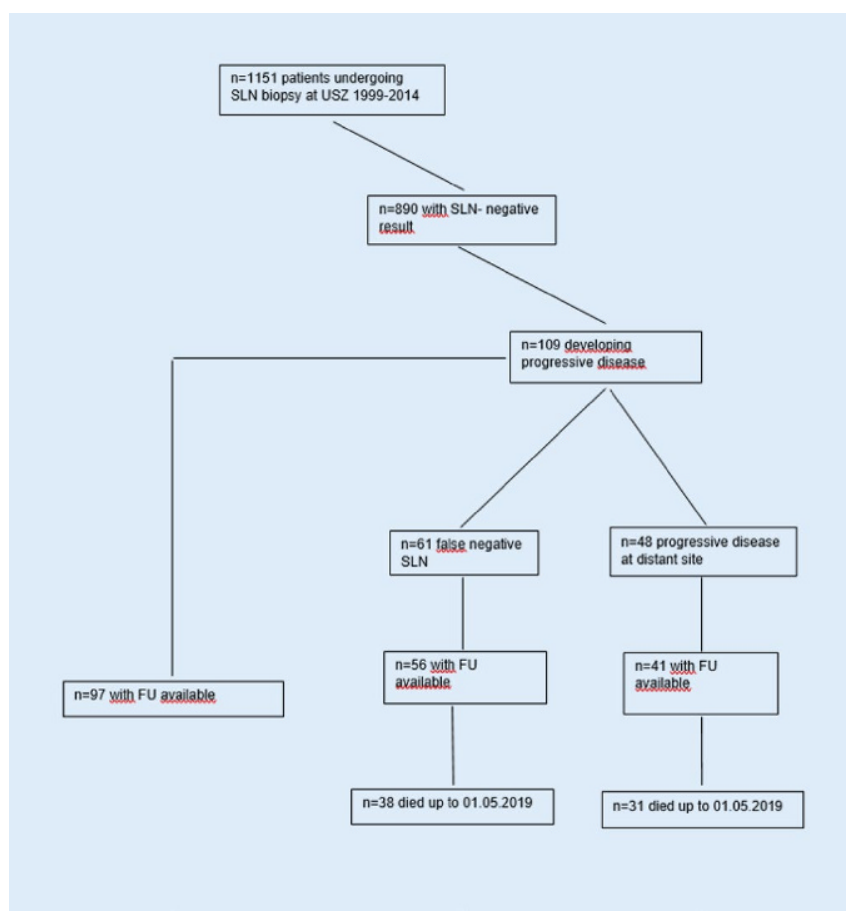

Fig. 1 | P 9 | 


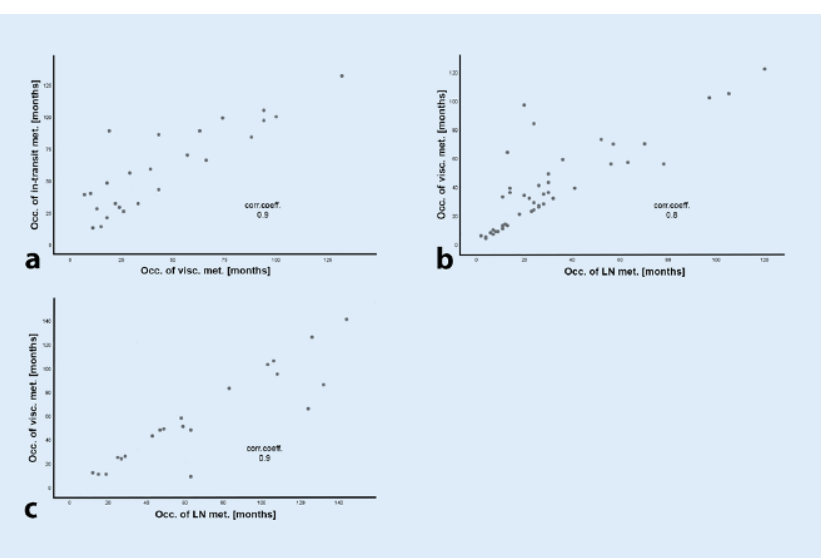

Fig. 2 |P 9 A

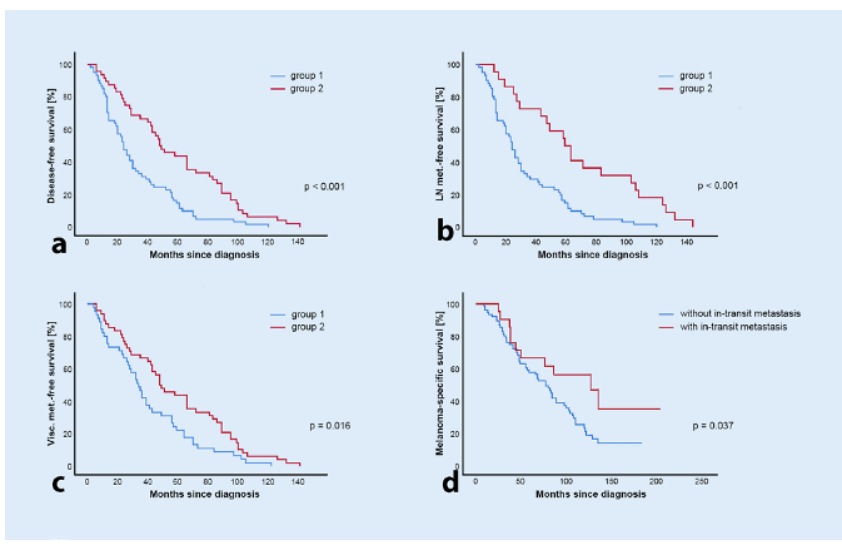

Fig. 3 | P 9 A

\section{P 10}

\section{Diffuse pulmonary meningotheliomatosis. A case report of a rare} disease

Roland Zweifel ${ }^{2 *}$, Britta Hartmann ${ }^{2}$, Sebastian Rusch ${ }^{2}$, Franco Gambazzi ${ }^{3}$, Rainer Grobholz

'Institut für Pathologie, Kantonsspital Aarau, Aarau, Switzerland; ${ }^{2}$ Institut für Labormedizin, Kantonsspital Aarau, Aarau, Switzerland; ${ }^{3}$ Klinik für
Thoraxchirurgie, Kantonsspital Aarau, Aarau, Switzerland

Background: Diffuse pulmonary meningotheliomatosis (DPM) is a rare condition. We report a case with review of the literature.

Methods: 72-year old woman with rheumatoid arthritis, exertional dyspnea and multiple bilateral centrilobular distributed noduli underwent surgical biopsy in order to prove an interstitial lung disease. Immunohistochemical studies and Next Generation Sequencing were performed.

Results: Histology revealed lung tissue with multiple small nodules of epitheloid cells with round to oval nuclei devoid of atypia and eosinophilic cytoplasm, mainly distributed in perivenular location and along the walls of alveolar septae. The nodules measured up to $5 \mathrm{~mm}$ in greatest dimension and demarcated sharply from the adjacent pulmonary parenchyma. Immunohistochemical studies showed a positivity of the tumor cells for epithelial membrane antigen, progesterone receptor and CD56. There was a negative staining for smooth muscle actin, CD34, CD117, S100 HMB45, AE1/3, Synaptophysin and Chromogranin A. The proliferation marker Ki-67 was $2 \%$.

Next Generation Sequencing showed gene mutations of $\mathrm{NOTCH} 2, \mathrm{MSH} 2$ and SETD2.

Conclusions: DPM is a rare entity which can clinically and histologically lead to misinterpretation of other diseases. Initially described as chemodectomas, subsequent ultrastructural and immunohistochemical studies demonstrated the characeristics of meningothelial cells. Our immunhistochemical results confirm previous reports of meningothelial differentiation in DPM.

To our knowledge this is the first case of DPM described in the context of rheumatoid arthritis and gene mutations of NOTCH2, MSH2 and SETD2. We discuss the controversial nature of DPM.

\section{P 11}

Impact of extracellular mucin detection in colorectal cancer using a novel unsupervised deep learning method

Huu Giao Nguyen ${ }^{1 *}$, Annika Blank ${ }^{2}$, Heather Dawson ${ }^{2}$, Alessandro Lugli ${ }^{2}$, Inti Zlobec $^{3}$

'Universität Bern, Bern, Switzerland; ${ }^{2}$ Institute of Pathology, University of Bern, Bern, Switzerland; ${ }^{3}$ Translational Research Unit, University of Basel, Basel, Switzerland

Background: Mucinous colorectal cancers are defined as containing $>50 \%$ extracellular mucin and are found in approximately $12 \%$ of all cases. This cut-off is, however, arbitrary and the clinical relevance of mucin is controversial. The aim of this study was to develop a deep learning algorithm

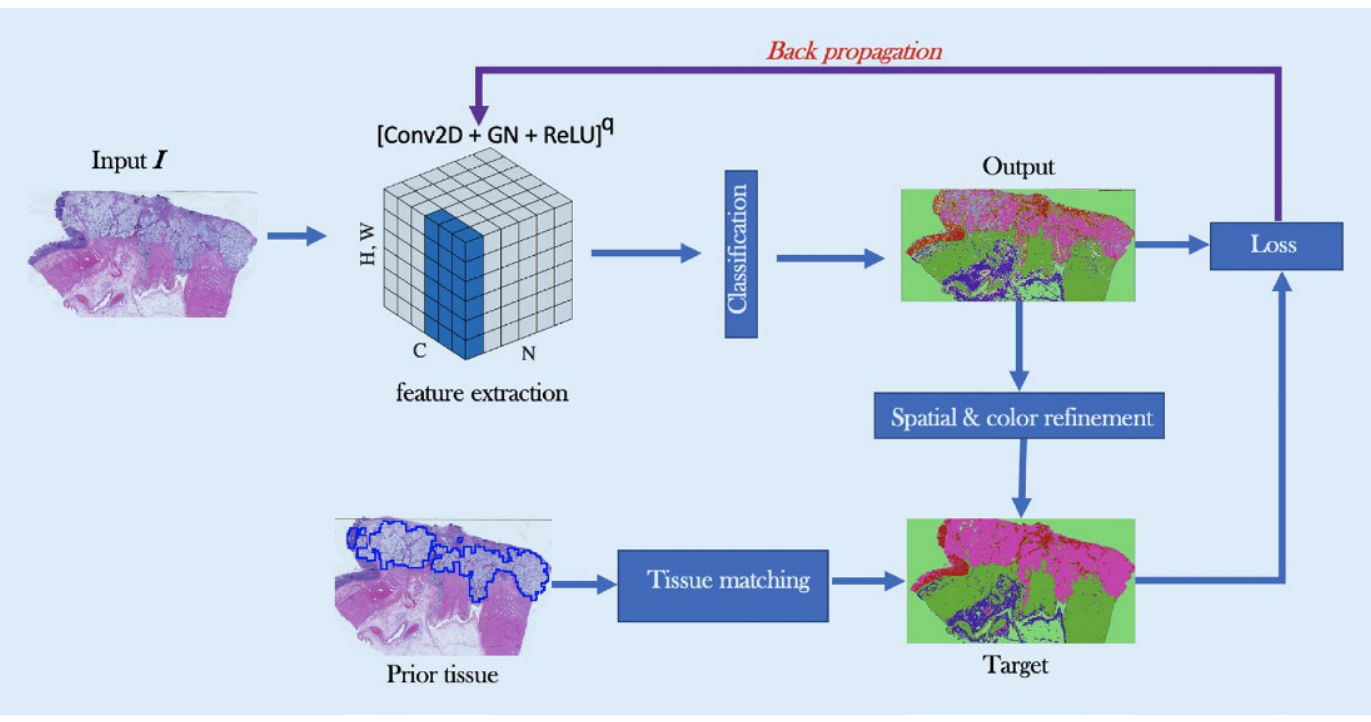

Fig. $1 \mid$ P 114 
for quantification of the percentage of mucin within the tumor area and to determine the association of mucin with clinicopathological features. Methods: We scanned 904 slides from 452 cases. Histological subtype and clinicopathological features were extracted from reports. We developed an unsupervised segmentation method to detect the percentage of extracellular mucin/tumor area by assigning a semantic label to each pixel in images and classifying them. The proposed method, namely Group Affinity Unsupervised (GAUS) learning has three main steps (• Fig. 1): 1) Forward process to create an output image based on the classification of $\mathrm{CNN}$ visual features 2) Creation of a target image from the similarity group matching of color, spatial coordinate and prior tissue pattern 3) Backpropagation process with a loss function to train and update the network parameters. Inter-observer agreement between algorithm and pathologists' scores was tested on 163 slides.

Results: - Fig. 2 shows an example of segmentation output. Inter-observer agreement between pathologists and algorithm was excellent ( $\bullet$ Fig. 3; ICC $=0.917)$. Mucinous histology ( $>50 \%$ mucin $)$ was associated with larger tumor size $(p=0.008)$, right-sided tumor location $(p=0.0428)$, more advanced pT $(p=0.002)$, less venous invasion $(p=0.0451)$, less tumor budding ( $p=0.023)$ and high levels of microsatellite instability (MSI-H) $(p<0.0001)$. The presence of any intratumoral mucin $(>0 \%)$ correlated with BRAF mutation $(p=0.0329)$.

Conclusions: We introduced an effective method for the mucin quantification in colorectal cancers, taking advantage of unsupervised learning without training data or pre-trained network. Moreover, tumors with any extracellular mucin behave similarly to cancers with mucinous histology ( $>50 \%$ mucin). A more comprehensive investigation into the topic of mucin in colorectal cancer is warranted.

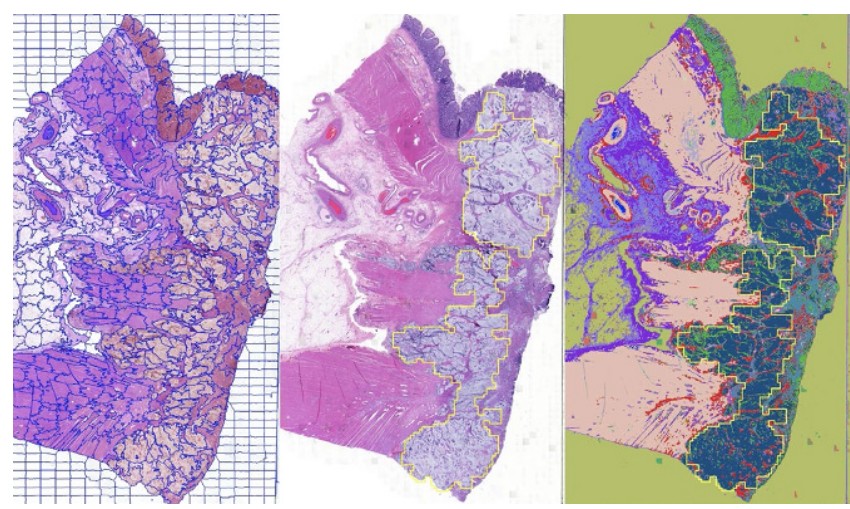

Fig. 2 |P 11 A

\begin{tabular}{|l|l|c|}
\hline \multicolumn{2}{|c|}{ ICC indexfor 3 raters } & 0.917 \\
\hline \hline Pearson correlation & GAUS vs. Pathologist 1 & 0.915 \\
\cline { 2 - 3 } & GAUS vs. Pathologist 2 & 0.917 \\
\cline { 2 - 3 } & Pathologist 1 vs. Pathologist 2 & 0.924 \\
\hline
\end{tabular}

Fig. $3 \mid$ P 11 A

\section{P 12}

Postmortem pulmonary pathology and virus detection in patients with COVID-19 infection: single-center report of 12 consecutive autopsies from Lausanne, Switzerland

Sabina Berezowska*, Karine Lefort, Kalliopi loannidou, Daba Ndiaye, Konstantinos Petrovas, Chun-Yi Tsai, Dina Milowich, Eleonore Multone, Nathalie Sala, Bettina Bisig, Laurence de Leval

Deparment of Laboratory Medicine and Pathology, Institute of Pathology, Lausanne University Hospital and Lausanne University, Lausanne, Switzerland

Background: The novel severe acute respiratory syndrome coronavirus 2 (SARS-CoV-2) pandemic has caused $>850,000$ deaths worldwide, with a disproportionate lack of autopsy studies.

Methods: We report postmortem pulmonary findings focusing on histology and virus detection in 12 of 14 consecutive patients who died with confirmed COVID-19. Two patients declined participation in research. SARS-CoV-2 virus detection in formalin-fixed paraffin embedded tissue was performed by reverse transcriptase quantitative PCR (RT-qPCR) and in-situ RNA hybridization (RNAscope).

Results: The 5 women and 7 men (median age: 73 years; range 36-96) died 3-38 days after onset of symptoms (median: 14.5 days). Eight patients received mechanical ventilation for 2-19 days.

Eleven patients showed diffuse alveolar damage (DAD) in different stages of evolution: 1 in early exudative phase, with congestion, edema and hyaline membrane formation; 6 (50\%) in late exudative phase, with additional thickening of alveolar septa and interstitial lympho-plasmacellular infiltrates; $4(33 \%)$ as proliferative phase/organizing DAD, in one patient presenting as acute fibrinous and organizing pneumonia. Acute bronchopneumonia was present in 6 patients $(50 \%)$, in one case without underlying $\mathrm{DAD}$. One patient showed invasive aspergillosis with necrotic bronchitis. Microthrombi were present only in exudative DAD in 5 patients. True necrotizing vasculitis was absent.

RT-qPCR detected high amounts of virus in 6 patients (50\%) with exudative $\mathrm{DAD}$ and symptom-duration $\leq 14$ days, supported by in-situ visualization of viral RNA in 3/3 cases analyzed. The 6 patients with borderline low viral copy levels were symptomatic for $\geq 15$ days, comprising all 4 cases with organizing DAD, the patient without DAD and one case of exudative DAD.

Conclusions: We show the high prevalence of DAD as a reaction pattern in COVID-19, the high number of overlying acute bronchopneumonia, and convincing pulmonary virus detection limited to patients who died $\leq 2$ weeks after onset of symptoms, correlating with pre-organization phases of DAD.

\section{P 13}

Metastases in hepatocellular carcinoma retain morphology but show difference in methylation profile

Simon Haefliger ${ }^{1 *}$, Colm Rourke ${ }^{2}$, Jesper Andersen ${ }^{2}$, Matthias S. Matter ${ }^{1}$ 'Department of Pathology, Institute of Pathology and Medical Genetics, University Hospital of Basel, Basel, Switzerland; ${ }^{2}$ Biotech Research and Innovation Centre (BRIC), University of Copenhagen, Copenhagen, Denmark

Background: Metastasis is the leading cause of death in cancer patients. Especially in hepatocellular carcinoma (HCC), the prognosis is poor once metastases have formed. Little is known about the mechanisms of metastasis formation in liver cancer and about the changes that occur from the primary tumor to metastasis. The aim of our study was therefore to gain insight into the similarities and differences between primary tumor and metastasis in patients with hepatocellular carcinoma.

Methods: We analyzed morphology between primary tumor $(n=24)$ and matched metastasis present in various organs (e.g. lung, lymph node, or adrenal glands). In particular, we compared histological growth patterns (e. g. trabecular, solid or pseudoglandular), cytological characteristics, and differentiation grade. In addition, we compared methylation profile of several primary HCCs and their matched metastasis. 
Results: 20 out of 24 patients ( $83.3 \%$ ) showed identical morphology between primary tumor and matched metastasis. In four cases $(16.6 \%)$, the metastasis did not show the same morphology as the primary tumor, for example the metastasis contained only one histological growth pattern whereas the primary tumor contained various growth patterns. In contrast, methylation analysis displayed a high degree of intra-donor clonality and exhibited patterns of divergent evolution. In particular, metastasis-associated fibroblasts showed the greatest extent of epigenetic reprogramming. Conclusions: Our study revealed a high concordance of morphology but pronounced differences in methylation profile between primary and matched metastasis of HCC patients.

\section{P 14}

Posttransplant EBV-negative diffuse large B-cell lymphoma with microsatellite instability: an unexpected finding revealed by NGS

Nicolas Dattner ${ }^{\prime *}$, Edoardo Missiaglia' ${ }^{1}$, Audrey Letourneau', Manuel Pascual', Delaviz Golshayan', Laurence de Leval', Bettina Bisig ${ }^{3}$

'Department of Laboratory Medicine and Pathology, Institute of Pathology; Lausanne University Hospital, Lausanne, Switzerland; ${ }^{2}$ Transplantation Center, Lausanne University Hospital and Lausanne University, Lausanne, Switzerland; ${ }^{3}$ Department of Laboratory Medicine and Pathology, Institute of Pathology; Lausanne University Hospital and Lausanne University, Lausanne, Switzerland

Background: Microsatellite instability (MSI) is very rare in lymphoid neoplasms, but may occur in immunodeficiency-related lymphomas such as posttransplant lymphoproliferative disorders (PTLD). A compromised immunity might indeed fail to counter the development of hypermutated tumours, despite numerous neoantigens. Moreover, some immunosuppressive treatments like azathioprine are thought to induce epigenetic silencing of mismatch repair (MMR) enzymes. We present a case of PTLD with MSI revealed fortuitously by NGS.

Methods: A customized 54-gene-NGS panel, designed for B-cell lymphomas and covering a total of $134 \mathrm{~kb}$, was applied to a PTLD recurrence. A mutational signature was determined based on the mutation patterns. MS status and MMR proteins were further explored by PCR and immunohistochemistry (IHC).

Results: A 1983-born man underwent renal transplantation in 1998 for post-meningococcemic renal failure, pancreatic allograft in 2011 for type 1 diabetes, and suffers from a severe Crohn disease since 2013. He has received several immunosuppressive treatments including corticoids, calcineurin inhibitors, mycophenolate and azathioprine. In August 2019, gastric and pulmonary biopsies revealed an EBV-negative diffuse large B-cell lymphoma-type PTLD. Despite chemotherapy, a gastric PTLD recurrence was diagnosed in January 2020. NGS, performed to search for potential treatment targets, identified numerous non-synonymous mutations (likely equivalent to a tumour mutation burden $>100 / \mathrm{Mb}$ ), mostly transitions and small indels in homopolymeric sequences, suggestive of an MSI-associated mutational signature. MMR IHC showed a complete loss of MSH2 expression and a partial loss of MSH6. PCR confirmed MSI (5 instable markers/5).

Conclusions: Although small NGS panels are not considered suitable to determine a mutational signature, particular mutation patterns may hint towards a specific etiopathogenesis such as MSI, opening up new treatment opportunities. Reduction of immunosuppression and/or immune checkpoint inhibitors could theoretically have restored immune recognition of this hypermutated PTLD, but were contraindicated because of the patient's comorbidities. He is currently responding to polatuzumab (antiCD79b)-bendamustine.

\section{P 15}

\section{Automatic detection of $\mathbf{h}$.pylori in gastric biopsies*}

Tim Ogi, Inti Zlobec, Huu Giao Nguyen, Bastian Dislich

University of Bern, Institute of Pathology, Bern, Switzerland

Background: Infection with Helicobacter pylori is commonly diagnosed in Giemsa-stained slides from gastric biopsies. This diagnostic task occurs daily and can be challenging and time-consuming. Here, we sought to au-

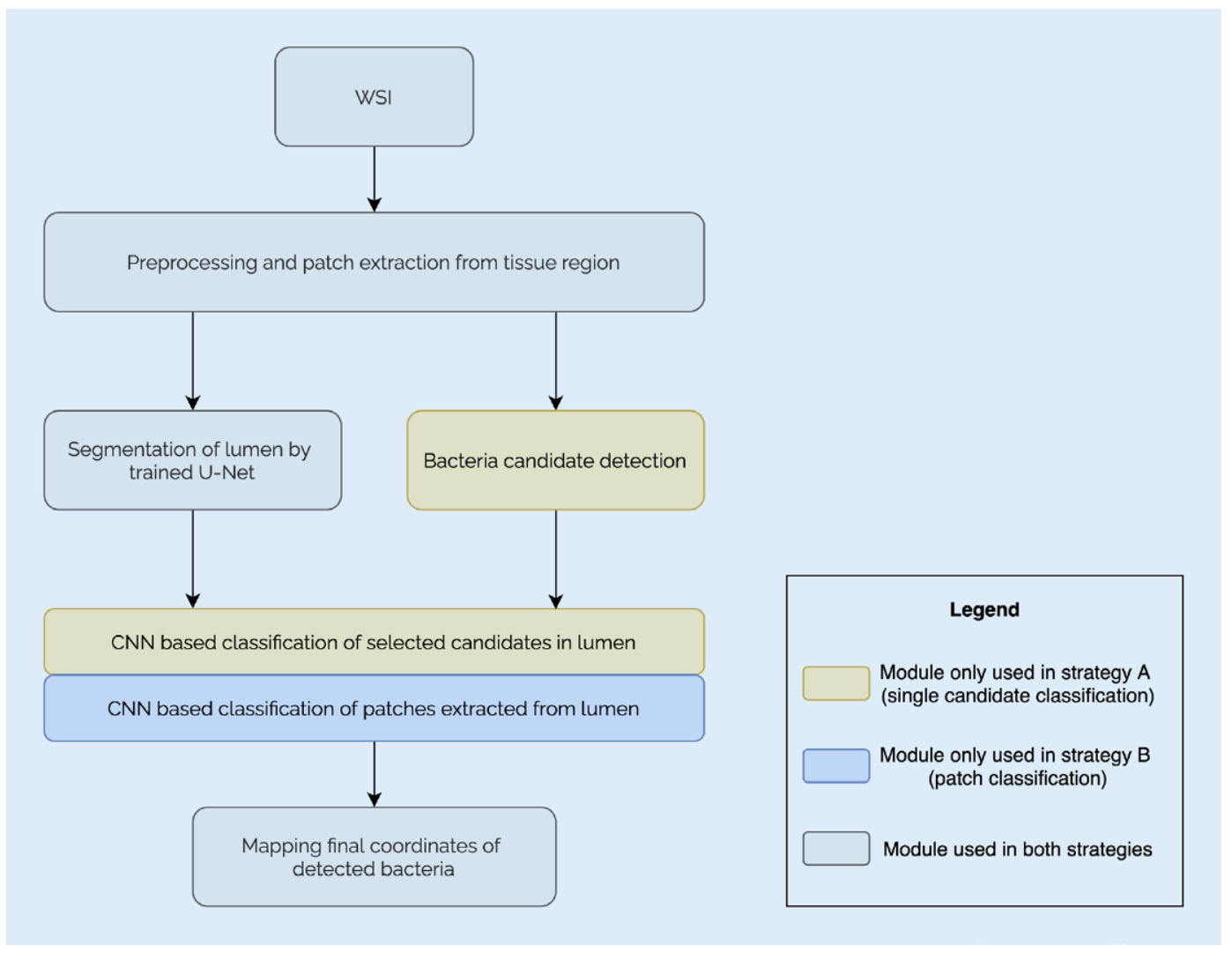

Fig. $1 \mid \mathrm{P} 15<$ 
tomate the detection of $H$. pyloriin whole slide images of Giemsa-stained gastric biopsies using deep learning methods.

Methods: 41 slides from different patients were scanned using a Hamamatsu S360 slide scanner at $40 \times$ magnification (resolution: $0.23 \mu \mathrm{m} / \mathrm{pixel}$ ). The lumen region, where $H$. pyloriis commonly found, was initially segmented using a U-Net convolutional neural network (CNN), which had been trained with 1048 annotated images. Subsequently, another CNN assessed the presence of $H$. pylori in patches extracted according to one of two strategies we evaluated: A) Laplacian of Gaussian blob detection was used to identify patches containing objects of the same size as $H$. pylori and B) patches were extracted directly from the lumen region using a grid (- Fig. 1). We compared the three state-of-the-art CNN architectures MobileNetV2, ResNet50V2, and Xception trained on a dataset of 754 images, created by an expert pathologist.

Results: $98 \%$ of lumen regions were detected by the U-Net, the dice overlap index was $0.89 \pm 0.07$ (• Fig. 2), and the search space was narrowed down to $3.7 \pm 2.3 \% .99 \%$ of all bacteria were annotated as candidates by blob detection. The best performing architectures were Xception in strategy B $(\mathrm{F} 1=0.989 \pm 0.009)$, and ResNet50V2 $(\mathrm{F} 1=0.859 \pm 0.053)$ in strategy A. The assembled pipeline with strategy A/B detected $26 \% / 35 \%$ truepositive, $25 \% / 37 \%$ false-positive, and $49 \% / 28 \%$ objects that could not be classified by our pathologist.

Conclusions: Despite the high number of false-positive results, we succeeded in demonstrating that it is possible to automatically detect $H$. $p y$ lori in gastric biopsies. Our results may improve with a larger database of annotated $H$. pylori images and higher-resolution scans.

* Student paper

\section{P 16}

Superior survival prediction using a combined score of major pathological response, ypT and ypN compared to the standard TNM model in non-small cell lung cancer after neoadjuvant therapy*

Philipp Zens ${ }^{1 *}$, Corina Bello', Amina Scherz ${ }^{2}$, Julia Koenigsdorf', Alexander Pöllinger ${ }^{3}$, Ralph A. Schmid', Adrian Ochsenbein ${ }^{2}$, Christina Neppl', Rupert Langer ${ }^{5}$, Sabina Berezowska ${ }^{6}$

Institute of Pathology, University of Bern, Bern, Switzerland; ${ }^{2}$ Department of Medical Oncology, Inselspital University Hospital Bern, Bern, Switzerland; ${ }^{3}$ Department of Diagnostic, Interventional and Pediatric Radiology, Inselspital University Hospital Bern, Bern, Switzerland; ${ }^{4}$ Department of General Thoracic Surgery, Inselspital University Hospital Bern, Bern, Switzerland; 'Institute of Pathology, Kepler Universitätsklinikum, Linz, Austria; ${ }^{6}$ Deparment of Laboratory Medicine and Pathology, Institute of Pathology, Lausanne University Hospital and Lausanne University, Lausanne, Switzerland

Background: The current standard prognostic models in non-small cell lung cancer patients after neoadjuvant treatment are the TNM classification and major pathological response (MPR) with a cut-off of $10 \%$ residual tumor. We aimed to integrate tumor regression in a prognostic score (PRSC) to improve the possibility of clinical decision making based on a TNM and morphology-based system with increased predictive power. Methods: 117 consecutive patients resected after neoadjuvant therapy between 2000 and 2016 were included in this monocentric retrospective study. During histopathological re-evaluation, the amount of residual tumor in the primary tumor bed was assessed according to the recommendations of the IASLC and tumor regression was graded into five categories: $0 \%,<1 \%, 1-10 \%, 11-49 \%$ and $\geq 50 \%$ residual tumor. After confirming the prognostic significance of the TNM staging system and tumor regression, we included ypT, ypN and major pathological response (MPR) in a PRSC generating a three-tier classification. Cox-proportional hazard models were used for univariate and multivariate analyses. The Akaike and Bayesian Information Criterion were used to compare the goodness-of-fit. Results: The isolated ypT-categories $(p<0.001, p<0.001)$ and combined TNM8 stages $(p=0.004, p=0.013$ ) had significant impact on overall (OS) and disease-free survival (DFS) stratification. We confirmed the importance of histologyspecific interpretation of MPR confirming the cut-offs at 65 and $10 \%$ residual tumor in adenocarcinoma and non-adenocarcinoma, respectively, as previously reported. MPR significantly stratified OS $(p=0.01)$ and DFS $(p<0.001)$. The PRSC significantly stratified a low-, in-

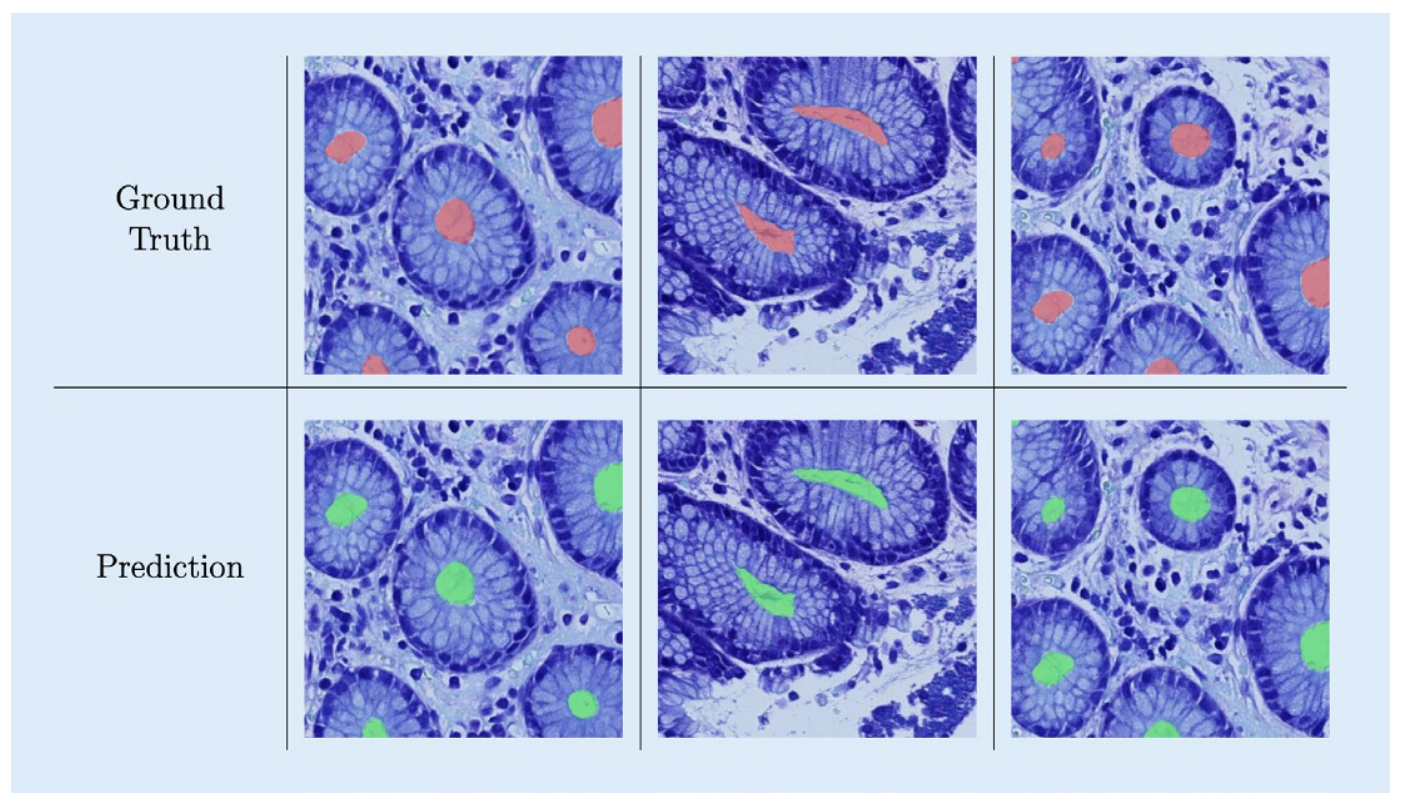


termediate- and high-risk group ( $p<0.001$ for OS/DFS). The PRSC yielded a better fitting model compared to the TNM classification or MPR alone. Conclusion: We established a prognostic score with the potential to more accurately predict patient survival, which is readily applicable in routine diagnostics.

* Student paper

\section{P 17}

Significance of tumor regression in lymph node metastases of esophageal adenocarcinomas after neoadjuvant therapy *

Marek Michal Osecky ${ }^{1 *}$, Rupert Langer ${ }^{2}$, Bastian Dislich ${ }^{3}$, Karen Becker', Marcus Feith ${ }^{5}$, Dino Kröll ${ }^{6}$

'Institute of Pathology, University of Berne, Berne, Switzerland; 'Institute of Pathology and molecular Pathology, University of Bern, Bern,

Switzerland; ${ }^{3}$ University of Bern, Institute of Pathology, Bern, Switzerland; ${ }^{4}$ Institute of Pathology, Technische Universität München, Munich,

Germany; ${ }^{5}$ Department of Surgery, Klinikum rechts der Isar, TUM School of Medicine, Munich, Germany; ${ }^{6}$ Department of Visceral Surgery and Medicine, Inselspital, Bern University Hospital and University of Bern, Bern, Switzerland.

Background: Tumor regression following neoadjuvant treatment can be observed in lymph node (LN) metastases similar to the primary tumor in esophageal adenocarcinomas (EAC). We evaluated the prognostic significance of tumor regression in LN metastases locally advanced EAC following neoadjuvant treatment.

Methods: $239 \mathrm{EAC}$ patients treated with neoadjuvant radiochemotherapy (RCTX) or chemotherapy (CTX) followed by esophagectomy were analyzed. We examined retrospectively the $\mathrm{LN}$ for histopathologic signs of regression, i. e. nodular fibrosis and acellular mucin. LN classification was performed according to two parameters: presence $(-)$ or absence $(+)$ of residual tumor and regression characteristics in the $\mathrm{LN}$, resulting in four categories: LN-/REG-, LN-/REG+, LN+/REG+, LN+/REG-.

Results: LN metastases with residual tumor were detectable in 117/239 (49\%) cases. Regression in LN were observed in 85/239 cases (35.5\%). The distribution of the LN/REG categories were as follows: 97 patients (40.6\%) were LN-/REG-. 25 patients (10.5\%) were LN-/REG+. 60 (25.1\%) were $\mathrm{LN}+/ \mathrm{REG}+$ and 57 (23.8\%) LN+/REG-. The LN/Reg categorization had a significant prognostic value in univariate analysis $(p<0.001)$ and multivariate analysis $(\mathrm{HR}=1.326 ; p=0.002)$ with similar results for the subgroups of patients treated with RCTX or CTX. The prognosis of LN-/REG+ was worse than LN-/REG- but better than both LN+ categories, which was clearly seen in the Kaplan Meier curves but did not reach statistical significance ( $p=0.104$ and $p=0.090$, respectively). In contrast, there was no difference between LN+/REG+ and LN+/REG- $(p=0.802)$.

Conclusions: Regression in LN metastases of EAC can be observed in a significant number of patients after neoadjuvant therapy. The most relevant negative prognostic factor is the presence of LN metastases, independent of the degree of regression. Complete regression of former LN metastases in comparison to "true" negative LN seems to be of prognostic relevance but additional studies are needed to confirm this trend seen in our study. * Student paper

\section{P 18}

\section{Immunohistochemistry for SARS-CoV-2 in autoptic pulmonary tissue}

Nathalie Schwab ${ }^{1 *}$, Ronny Nienhold ', Haslbauer Jasmin ${ }^{2}$, Melanie Sachs', Niels Willi', Matthias S. Matter ${ }^{2}$, Alexandar Tzankov', Kirsten Mertz', Gieri Cathomas $^{4}$

'Institute of Pathology, Cantonal Hospital Baselland, Liestal, Switzerland; ${ }^{2}$ Institute of Medical Genetics and Pathology, University Hospital Basel, University of Basel, Basel, Switzerland; ${ }^{3}$ Institute of Medical Genetics and Pathology, University Hospital Basel, Basel, Switzerland; ' ${ }^{4}$ nstitute of Pathology, Cantonal Hospital Baselland, Liestal, Switzerland
Introduction: Covid-19 is primarily a disease of the lungs. SARS-CoV-2, the virus causing the disease, is usually detected by PCR-based methods. In contrast, little is known about the value of immunohistochemistry (IHC) for visualizing SARS-CoV-2 in the tissue.

Materials and methods: Pulmonary FFPE autopsy tissue of patients with confirmed Covid-19 infection was analyzed by IHC using an anti-SARSCoV nucleocapsid (N) antibody. In addition, the copy number of the viral RNA was determined by quantitative RT-PCR, which targets three genomic regions specific for SARS-CoV-2 and the human control gene RNaseP. Results: 88 pulmonary samples of 18 Covid-19 patients were analyzed. IHC was positive in $63(71.6 \%)$ samples of $10(55.6 \%)$ patients and RNA detected in $83(94.3 \%)$ tissues of $16(88.9 \%)$ patients. IHC revealed a heterogeneous distribution of positive cells and stained primarily pneumocytes and alveolar macrophages and only rarely other cells such as bronchial epithelium. Quantitative analysis of SARS-CoV-2 RNA showed a significant difference between IHC positive and negative samples (101,348 viral copies per $106 \mathrm{RNaseP}$ molecules vs $123, p<0.005$ ). In concordance with this finding, a wide range of viral RNA was detected in different tissue samples of each individual patient. Patients positive by IHC showed a significantly shorter duration between the diagnosis of Covid-19 to death as well as shorter hospitalization times (5.4 days vs 12.9 , and 3.3 vs 13.0 , $p<0.05$, respectively).

Discussion: IHC can be applied to pulmonary tissue to detect SARS-CoV-2. The strong correlation between RNA load and positive IHC indicates IHC being less sensitive. However, the fact that patients with positive IHC have a significantly shorter duration of disease indicates a biological difference and supports the concept of two phases of Covid-19, an early phase driven primarily by the virus and a late phase driven by other mechanisms.

\section{P 19}

\section{Effects of multiple scanners on deep learning based lymph node} segmentation in histopathology images *

Amjad Khan ${ }^{1 *}$, Annika Blank', Felix Müller', Huu Giao Nguyen ', Alessandro Lugli', Heather Dawson', Jean-Philippe Thiran'2, Inti Zlobec'

${ }^{1}$ Institute of Pathology, University of Bern, Bern, Switzerland; ${ }^{2}$ École polytechnique fédérale de Lausanne, Lausanne, Switzerland

Background: Stain color and contrast variations are common challenges faced by digital analysis of whole slide images (WSI) when glass slides are scanned with different scanners in an institute of pathology. These variations can further affect the outcome of computational pathology in daily diagnostic practice. We propose a novel study to assess the effects of scanner variability on lymph node segmentation.

Methods: Four different scanners were used to scan the same one hundred glass slides of lymph node tissues stained with hematoxylin and eosin. Two experienced pathologists annotated all four hundred WSI by manually drawing polygons around each lymph node. We applied a U-Net based lymph node segmentation pipeline with crossvalidation. To avoid overfitting, training samples were augmented by flipping, rotating, shearing, zooming, and cropping. Finally, segmented regions were quantitatively evaluated with annotations by DSC (Dice Similarity Coefficient) on a scale between zero (no overlap) and one (100\% overlap). Similarly, HD (Hausdorff Distance) measures the boundary loss on lymph node capsule where zero HD means no loss.

Results: In a first analysis, the trained model on one scanner was used to predict lymph node regions on test sets of all scanners. Variability was evident from the outcome, where Scannerl (DSC: $0.330 \pm 0.261,0.153 \pm 0.141$, $0.124 \pm 0.115$ and HD: $6.717 \pm 2.636,8.402 \pm 2.739,9.561 \pm 2.316)$ and Scanner4(DSC: $0.533 \pm 0.314$ and HD: $7.743 \pm 3.481$ ) showed poor performance. In a second analysis, pre-trained weights of one scanner were used to fine-tune other scanners. Fine-tuning minimized the variations of the results observed in the first analysis, where Scanner 1 (DSC: $0.865 \pm 0.214$, $0.842 \pm 0.243,0.856 \pm 0.219$ and HD: $3.128 \pm 1.312,3.378 \pm 1.534$, $3.273 \pm 1.412$ ) and Scanner4 (DSC: $0.877 \pm 0.156$ and HD: $4.802 \pm 2.630$ ) showed improvement. The above experiments were repeated for all four scanners simultaneously. 
Conclusions: Our analysis indicates that scanner variability can be optimized by fine-tuning. This technique will be valuable for institutes of pathology using different scanner types.

* Student paper

\section{P 20}

\section{Self-supervised learning for weakly labelled data in colorectal cancer*}

Christian Abbet ${ }^{*}$, Linda Studer ${ }^{2}$, Inti Zlobec ${ }^{3}$, Behzad Bozorgtabar', JeanPhilippe Thiran

'École polytechnique fédérale de Lausanne, Lausanne, Switzerland; ${ }^{2}$ Université de Fribourg, Fribourg, Switzerland; ${ }^{3}$ Translational Research Unit, University of Basel, Basel, Switzerland

Background: With the recent expansion of digital pathology, the need for large scale labeled data has increased. However, data annotation is a tedious task that is both time-consuming and expensive as it involves pathologists experts. In this work, we show that we can benefit from large unlabelled cohorts to learn meaningful data representations and reduce the burden of costly annotation, closing the performance gap with supervised approaches in down-stream tasks.

Methods: We propose a self-supervised learning solution that relies on state-of-the-art momentum contrast visual data representation. The approach is based on contrastive learning that is able to discriminate between different tissue examples widely available in digital pathology. We validate our approach using two publicly available datasets of colorectal tissues that contain more than 100,000 labeled patches classified into 9 different tissue types.

Results: Firstly, we pre-train a model for each cohort without considering labels, e.i. in an unsupervised fashion.

Secondly, we apply a simple linear classifier on top of frozen features (or fine-tuned) to classify the different tissue types using only a fraction of the available labels (see tables). We compare the results with the standard supervised approach. We achieve better performance on one dataset $(+2.6-3.7 \%)$ and similar performance with the second on linear evaluation. Moreover, the approach achieves a better score for specific classes such as tumors $(+1.9-4.0 \%)$, lymphocytes $(+1.5-2.8 \%)$, normal mucosa $(+2.3-3.6 \%)$, or debris $(+3.2-5.4 \%)$.

Conclusions: In our work, we showed that we could take advantage of the large quantity of unlabeled data generated by scanners to learn embedding spaces, which encode the semantic structure of data. Moreover, by labeling just a small fraction of the data, we achieve similar performances as supervised approaches. This point is critical as it would eventually reduce the annotation time for pathologists while keeping similar sensitivity-specificity trends compared to the supervised models.

* Student paper

\begin{tabular}{lccrrrrrrr}
\hline \hline Methods & TUM & STR & COMP & LYM & DEB & NORM & ADI & BACK & Average \\
\hline Frozen: & & & & & & & & & \\
Supervised & 91.0 & $\mathbf{7 5 . 9}$ & 73.8 & 87.6 & $\mathbf{8 8 . 7}$ & 92.5 & $\mathbf{9 7 . 8}$ & $\mathbf{9 8 . 7}$ & 88.5 \\
Mom. Encoder (ours) & $\mathbf{9 4 . 2}$ & $\mathbf{8 5 . 3}$ & $\mathbf{8 0 . 2}$ & $\mathbf{9 0 . 6}$ & $\mathbf{9 1 . 0}$ & $\mathbf{9 8 . 4}$ & 97.1 & 98.2 & $\mathbf{9 2 . 0}$ \\
\hline $\begin{array}{l}\text { Fine-tuned: } \\
\text { Supervised }\end{array}$ & 95.1 & 84.9 & 84.1 & $\mathbf{9 3 . 6}$ & 93.6 & 97.6 & 97.3 & $\mathbf{9 8 . 7}$ & 93.2 \\
Mom. Encoder (ours) & $\mathbf{9 6 . 6}$ & $\mathbf{8 9 . 5}$ & $\mathbf{8 8 . 9}$ & 92.2 & $\mathbf{9 4 . 6}$ & $\mathbf{9 8 . 7}$ & $\mathbf{9 8 . 3}$ & $\mathbf{9 8 . 7}$ & $\mathbf{9 4 . 8}$ \\
\hline
\end{tabular}

Fig. 1 | P 20 ム Kather-16 $16^{1}$ Fl-score over classes (100\% labels) for tumor (TUM), stroma (STR), complex stroma (COMP), lymphocytes (LYM), debris (DEB), normal (NORM), adipose (ADI), background (BACK)

\begin{tabular}{lccrrrrrrrr}
\hline \hline Methods & TUM & STR & MUC & LYM & NORM & DEB & ADI & BACK & MUS & Average \\
\hline Frozen: & & & & & & & & & & \\
Supervised & 92.2 & $\mathbf{7 2 . 4}$ & 96.7 & 90.9 & 88.2 & 88.7 & $\mathbf{9 9 . 2}$ & 99.2 & $\mathbf{8 4 . 7}$ & $\mathbf{9 2 . 5}$ \\
Mom. Encoder (ours) & $\mathbf{9 5 . 0}$ & 50.8 & $\mathbf{9 7 . 0}$ & $\mathbf{9 7 . 8}$ & $\mathbf{9 2 . 2}$ & $\mathbf{9 1 . 3}$ & 96.9 & $\mathbf{1 0 0 .}$ & $\mathbf{7 8 . 0}$ & 92.0 \\
\hline Fine-tured: & & & & & & & & & & \\
Supervised & $\mathbf{9 7 . 6}$ & 71.0 & $\mathbf{9 8 . 5}$ & 98.0 & $\mathbf{9 3 . 7}$ & $\mathbf{9 8 . 0}$ & $\mathbf{9 6 . 5}$ & $\mathbf{1 0 0 .}$ & $\mathbf{7 8 . 9}$ & $\mathbf{9 4 . 4}$ \\
Mom. Encoder (ours) & 93.1 & $\mathbf{7 5 . 3}$ & 94.4 & $\mathbf{9 9 . 4}$ & 93.1 & 94.6 & 95.6 & $\mathbf{1 0 0 .}$ & $\mathbf{8 3 . 0}$ & 93.3 \\
\hline \hline
\end{tabular}

Fig. 2 | P $20 \Delta$ Kather-19' Fl-score over classes (100\% labels) for tumor (TUM), stroma (STR), mucin (MUC), lymphocytes (LYM), normal (NORM), debris (DEB), adipose (ADI), background (BACK), smooth muscle (MUS)

\begin{tabular}{|c|c|c|c|c|c|c|c|c|}
\hline \multirow[b]{2}{*}{ Methods } & \multicolumn{4}{|c|}{ Kather16 } & \multicolumn{4}{|c|}{ Kather19 } \\
\hline & $10 \%$ & $20 \%$ & $50 \%$ & $100 \%$ & $1 \%$ & $2 \%$ & $5 \%$ & $100 \%$ \\
\hline \multicolumn{9}{|l|}{ Frozen: } \\
\hline Supervised & 85.3 & 86.7 & 87.8 & 88.5 & 89.5 & 90.1 & 90.8 & 92.5 \\
\hline Mom. Encoder (ours) & 88.5 & 90.2 & 91.1 & 92.0 & 89.9 & 90.3 & 90.6 & 92.0 \\
\hline \multicolumn{9}{|l|}{ Fine-tuned: } \\
\hline Supervised & 88.2 & 89.7 & 91.9 & 93.2 & 92.4 & 93.0 & 93.4 & 94.4 \\
\hline Mom. Encoder (ours) & 85.9 & 89.4 & 92.0 & 94.8 & 91.8 & 92.3 & 92.8 & 93.3 \\
\hline
\end{tabular}

Fig. $\mathbf{3}$ | P $20 \Delta$ Fl-score over all datasets with different percentages of training labels. We highlight top scores under statistical relevance ( $p$-value $<0.05$ ) with respect to other models if only part a subset of labels are used 